\title{
Targeting prion-like protein doppel selectively suppresses tumor angiogenesis
}

\author{
Taslim A. Al-Hilal, ${ }^{1,2,3}$ Seung Woo Chung, ${ }^{1}$ Jeong Uk Choi, ${ }^{1}$ Farzana Alam, ${ }^{4}$ Jooho Park, ${ }^{1}$ Seong Who Kim, ${ }^{5}$ Sang Yoon Kim, ${ }^{2,6}$ \\ Fakhrul Ahsan, ${ }^{3}$ In-San Kim, ${ }^{2,7}$ and Youngro Byun ${ }^{1,4}$ \\ ${ }^{1}$ Research Institute of Pharmaceutical Sciences, College of Pharmacy, Seoul National University, Seoul, South Korea. ${ }^{2}$ Biomedical Research Institute, Korea Institute of Science and Technology, Seoul, \\ South Korea. ${ }^{3}$ Department of Pharmaceutical Sciences, School of Pharmacy, Texas Tech University Health Science Center, Amarillo, Texas, USA. ${ }^{4}$ Department of Molecular Medicine and Biopharmaceutical \\ Sciences, Graduate School of Convergence Science and Technology, Seoul National University, Seoul, South Korea. ${ }^{5}$ Department of Biochemistry and Molecular Biology, Asan Medical Center College of \\ Medicine, and ${ }^{6}$ Department of Otolaryngology, Asan Medical Center, College of Medicine, University of Ulsan, Seoul, South Korea. ${ }^{7}$ KU-KIST School, Korea University, Seoul, South Korea.
}

Controlled and site-specific regulation of growth factor signaling remains a major challenge for current antiangiogenic therapies, as these antiangiogenic agents target normal vasculature as well tumor vasculature. In this article, we identified the prion-like protein doppel as a potential therapeutic target for tumor angiogenesis. We investigated the interactions between doppel and VEGFR2 and evaluated whether blocking the doppel/VEGFR2 axis suppresses the process of angiogenesis. We discovered that tumor endothelial cells (TECs), but not normal ECs, express doppel; tumors from patients and mouse xenografts expressed doppel in their vasculatures. Induced doppel overexpression in ECs enhanced vascularization, whereas doppel constitutively colocalized and complexed with VEGFR2 in TECs. Doppel inhibition depleted VEGFR2 from the cell membrane, subsequently inducing the internalization and degradation of VEGFR2 and thereby attenuating VEGFR2 signaling. We also synthesized an orally active glycosaminoglycan (LHbisD4) that specifically binds with doppel. We determined that LHbisD4 concentrates over the tumor site and that genetic loss of doppel in TECs decreases LHbisD4 binding and targeting both in vitro and in vivo. Moreover, LHbisD4 eliminated VEGFR2 from the cell membrane, prevented VEGF binding in TECs, and suppressed tumor growth. Together, our results demonstrate that blocking doppel can control VEGF signaling in TECs and selectively inhibit tumor angiogenesis.

\section{Introduction}

The process of angiogenesis drives the progression and development of many diseases. Tumors, for example, advance from the dormant stage to highly aggressive and metastatic cancers as a result of angiogenesis (1). Current antiangiogenic drugs chiefly target VEGF and its receptor VEGFR2. Drugs that target the VEGF/VEGFR2 axis have been used to treat a number of cancers such as colorectal, renal, and metastatic breast cancers (2, 3). However, approved antiangiogenetic drugs indiscriminately target VEGF and VEGFR2 present in both healthy and cancerous cells and stifle cell-signaling pathways throughout the body $(4,5)$. Thus, there is a need for drugs that can selectively target pathological vasculatures but that do not concentrate over normal vasculatures. For angiogenesis-specific drug delivery, one clever approach involves disrupting VEGF/VEGFR2 signaling in a controlled and site-specific fashion.

We propose what we believe to be a novel approach to target a molecular marker that is altered and overexpressed selectively in tumor endothelial cells (TECs). Doppel, a prion-like protein, has recently been rediscovered as a TEC-specific surface marker with uncertain functions; doppel contains domains similar to those of cellular prions (PrP) and has a 25\% structural homology with $\operatorname{PrP}$

Conflict of interest: The authors have declared that no conflict of interest exists Submitted: June 25, 2015; Accepted: January 21, 2016.

Reference information: / Clin Invest. 2016;126(4):1251-1266. doi:10.1172/JCI83427.
(6). This protein is transiently expressed in the brain endothelium of neonates, but in adults, it is expressed only in testicular cells (7). Indeed, doppel-KO mice grow with no developmental defects except sterility in males $(8,9)$.

In this study, we observed that doppel was expressed in the vasculatures of both clinical and preclinical cancer samples, but not in normal endothelium, and its expression enhanced blood vessel formation. Unlike existing strategies to attack circulating growth factors, we sought to target the doppel-associated molecular pathways involved in tumor angiogenesis but spare the pathways involved in physiological angiogenesis. With this goal in mind, we hypothesized that doppel propagates tumor angiogenesis and that inhibition of doppel controls angiogenic signaling and reduces the extent of vessel formation in tumors.

To test our hypothesis, we chose to use a polysaccharide-based compound to target doppel. It has previously been shown that the sulfated glycosaminoglycans (GAGs) heparin and heparan sulfates function as ligands for prion proteins $(10,11)$. Although doppel does not contain the N-terminal octarepeat region of PrP, the main binding site of heparin, doppel has a globular domain that is hinged to a highly basic and arginine-rich flexible $\mathrm{N}$-terminal region of the cell surface (12). This structural orientation makes the TEC membrane highly positively charged, a chemical feature that would increase the avidity of negatively charged heparin-like GAGs to bind with doppel. On the basis of this assumption, we designed a new heparin-based compound (LHbisD4) by 
A

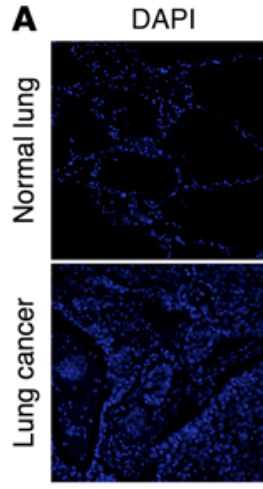

B

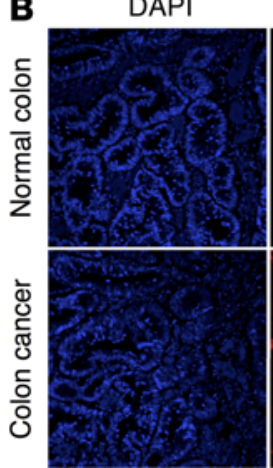

Doppel

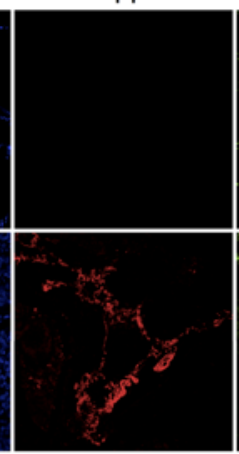

Doppel

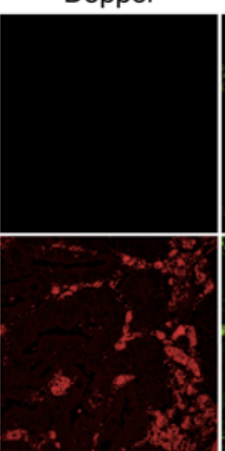

CD34

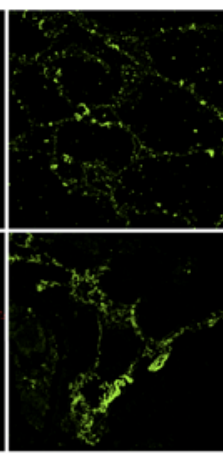

CD34

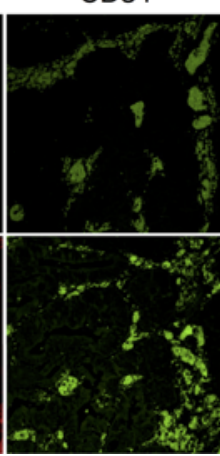

Merge

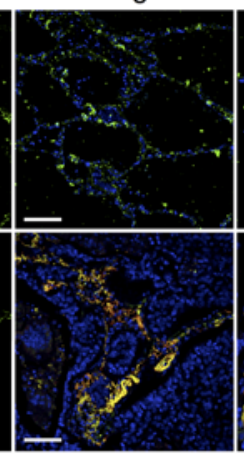

Merge

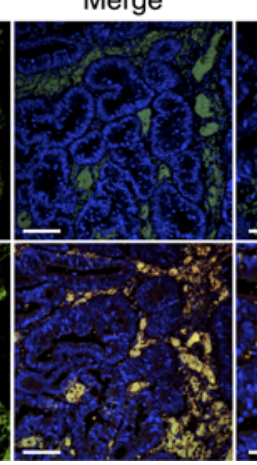

Magnified

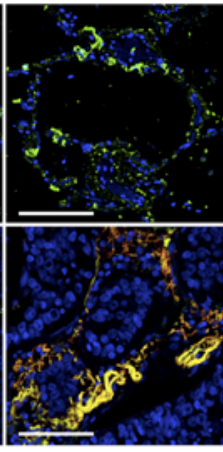

Magnified

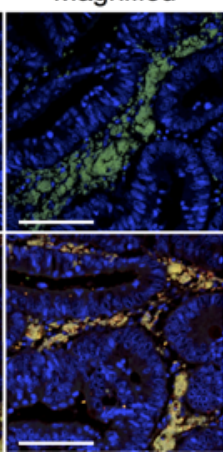

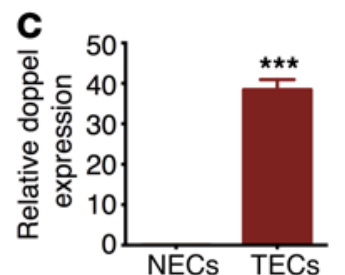

D $\mathrm{SCC} 7$ tumor $\mathrm{SCC} 7$ tumor
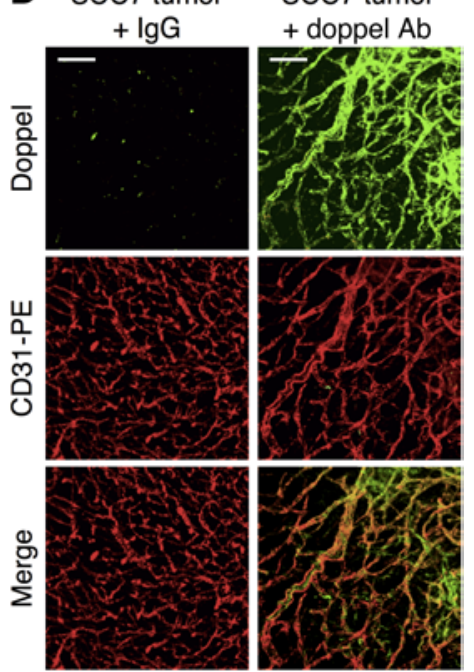

Figure 1. Expression of doppel in clinical and preclinical cancer tissues. Representative images of lung (A) and colon (B) tissues showing doppel (red), blood vessels (green; CD34), and nuclei (blue). Doppel colocalized with the blood vessels of cancer tissues, but not in normal tissues. Scale bars: $10 \mu \mathrm{m}$ (merge images) and $20 \mu \mathrm{m}$ (magnified images). $n=3-5$ tissues per group. (C) Relative mRNA expression levels of doppel in mouse NECs derived from brain tissue and TECs derived from SCC7 tumor (data represent 3 experiments). ${ }^{* *} P<0.001$ versus NECs, Student's $t$ test. (D) Whole-mount staining of SCC7 tumor section showing doppel expression (green) in tumor vessels (CD31). Note that incubation of control lgG failed to detect doppel in blood vessels of the tumor, confirming the specificity of the Ab and the accuracy of doppel detection. Scale bars: $50 \mu \mathrm{m}$. $n=5$ tumors.

conjugating heparin with deoxycholic acids (DOCA), which are large, rigid, polyhydroxylated steroidal acids that contain both multivalent and hydrophobic binding sites. DOCA conjugates can bind with ligands with superior affinity and avidity and facilitate oral availability (13-15). We posit that LHbisD4 will selectively target doppel and inhibit tumor angiogenesis. We believe that the enhanced specificity for targeting tumors, the high tumor-toorgan ratio, and oral delivery would constitute a more effective therapy than the current angiogenic therapies.

\section{Results}

Doppel is a tumor EC biomarker. We first evaluated the presence of doppel in human cancer tissues. For different commercially available Abs, immunofluorescence (IF) was performed to determine the specificity of the Ab to human doppel. The seminiferous duct cells of human testes showed strong reactivity to 3 Abs: rabbit anti-doppel Ab ( $\alpha$-doppel) (The Human Protein Atlas [HPA]); goat (G-20) $\alpha$-doppel; and goat (N-20) $\alpha$-doppel (both from Santa Cruz Biotechnology Inc.). However, the reactivity to rabbit (FL-176) $\alpha$-doppel (Santa Cruz Biotechnology Inc.) was rather weak (Supplemental Figure 1; supplemental material available online with this article; doi:10.1172/JCI83427DS1). Because the sensitivity and specificity of Abs vary from assay to assay, we reconfirmed the presence of doppel by Western blotting, which showed a single protein band with a molecular mass of approximately $42 \mathrm{kDa}$
(Supplemental Figure 2). TECs showed strong signals for doppel, but mouse brain ECs, doppel-knockdown TECs, and doppel-transfected HUVECs (Hu.dpl) did not signal for doppel, suggesting that the rabbit (FL-176) $\alpha$-doppel was specific to mouse doppel (Supplemental Figure 2A). Similarly, goat (N-20) $\alpha$-doppel was able to detect human doppel, because Hu.dpl signaled for doppel, but TECs, HUVECs, and doppel-knockdown Hu.dpl showed no signals for doppel. The application of PNGase F and neuraminidase to TECs produced bands with a small mass (Supplemental Figure 2B). Chemical deglycosylation using trifluoromethanesulfonic acid (TFMS) showed a band of approximately $15 \mathrm{kDa}$, suggesting that the doppel protein is remarkably and heterogeneously glycosylated in TECs (Supplemental Figure 2B). A similar glycosylation pattern was also observed for human doppel (16). Thus, IF is a valid method for the detection of doppel using goat (G-20) $\alpha$-doppel; the Western blot also validated the goat (N-20) $\alpha$-doppel and rabbit (FL-176) $\alpha$-doppel Abs against human and mouse doppel, respectively. Although independent analysis performed by the HPA showed no doppel expression in human cancer tissues, our study showed a differential expression pattern of doppel protein in clinical samples using the HPA and other commercially available Abs. Tissue sections containing primary human tumor (lung and colon) and normal tissues were used to assay doppel expression. In lung and colon cancer samples, doppel was strongly expressed and colocalized with CD34, a classical endothelial marker (Fig- 

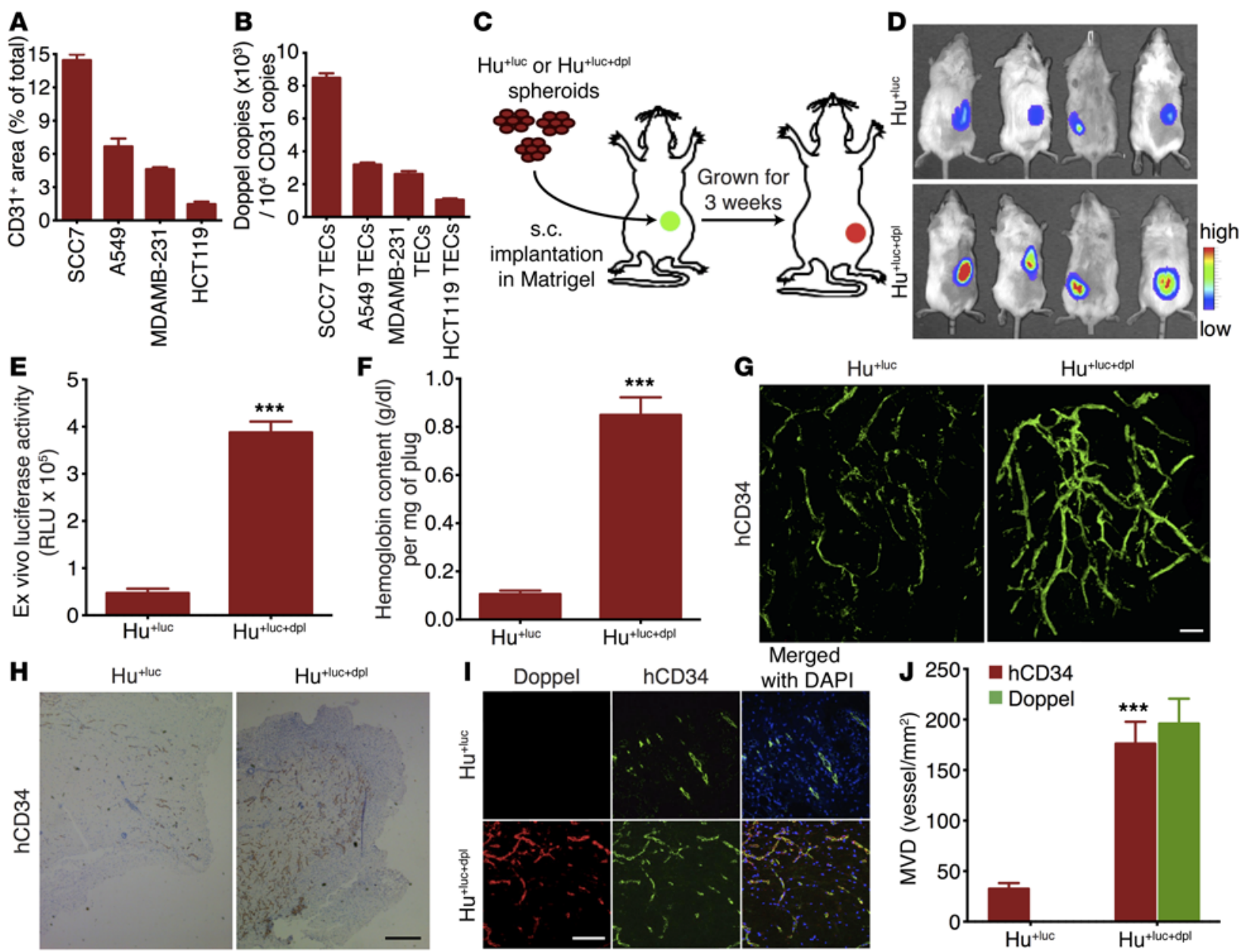

Figure 2. Increased doppel expression increases tumoral angiogenesis and EC function. (A) Total volume of blood vessels in squamous, lung, breast, and colon tumor. An aliquot of tumor ( $1 \mathrm{~g}$ ) was dissected to make single cells from the site where the CD31-positive area was calculated ( $n=3$ sections from each tumor). (B) Doppel expression in individual TECs as determined by flow cytometric analysis (3 experiments). (C) Experimental procedure for

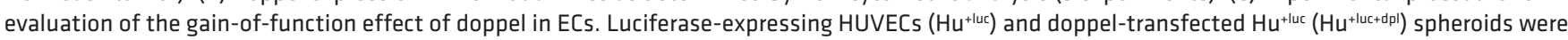
implanted s.c. in a Matrigel-fibrin matrix into female SCID mice. Three weeks after transplantation, the vascularization was analyzed. (D) Noninvasive monitoring of vascularization by bioluminescence imaging $\left(n=4\right.$ mice). (E) Ex vivo bioluminescence counts. ${ }^{* * *} P<0.001$ versus Hu+luc, Student's $t$ test. (F) Hemoglobin content within $\mathrm{Hu}^{+ \text {luc }}$ and $\mathrm{Hu}^{+ \text {luctdpl }}$ plugs was quantified. ${ }^{* * *} P<0.001$ versus $\mathrm{Hu}^{\text {+luc }}$, Student's $t$ test. (G) $3 D$ structure of the vascular net-

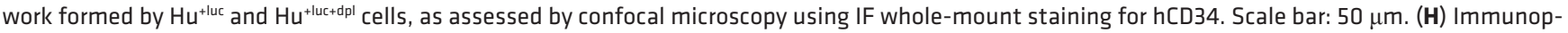
eroxidase detection of hCD34-positive blood vessels in $\mathrm{Hu}^{+ \text {tuc }}$ and $\mathrm{Hu}^{+ \text {tuctdpl }}$ plugs. Scale bar: $20 \mu \mathrm{m}$. (I) Characterization and images of vascular network by staining for doppel (red), hCD34 (green), and nuclei (blue) in Hu $\mathrm{Hu}^{\text {tluc }}$ and $\mathrm{Hu}^{\text {+luctdpl }}$ plugs. Scale bar: $20 \mu \mathrm{m}$. (J) Quantification of hCD34-positive and doppel-positive mean vessel density (MVD) in $\mathrm{Hu}^{+ \text {luc }}$ and $\mathrm{Hu}^{+ \text {luctdpl }}$ plugs. Doppel-positive vessels were not detected in $\mathrm{Hu}^{+ \text {luc }}$ plugs. ${ }^{* * *} P<0.001$ versus Hutluc, Student's $t$ test. $n=4$ plugs per experiment.

ure 1, A and B, and Supplemental Figure 3). IF images of tissue microarrays (TMAs) containing human cancer (18 non-small-cell lung cancers [NSCLCs] and 32 colon cancers), cancer-adjacent tissues (18 lung and 16 colon), and normal tissues (18 lung and 1 colon) were also evaluated for doppel expression. Despite some variability, $83 \%$ (15 of 18 samples) of NSCLC and 78\% (25 of 32 samples) of colon cancer samples, but not adjacent or normal tissues, showed increased doppel expression (Supplemental Figures 4 and 5). Doppel was predominantly found in cancer vasculatures. Colocalization of doppel with CD34 was confirmed by a Pearson coefficient of greater than 0.5 (Supplemental Figure 4C and Supplemental Figure 5C).

To evaluate doppel expression at the molecular level, we isolated TECs from the highly angiogenic squamous cell carcinoma
7 (SCC7) grown in mice (Supplemental Figure 6A). Transcription levels, determined by quantitative reverse transcription PCR (RT-PCR), were approximately 38-fold greater in TECs from SCC7 tumor than in the murine normal endothelial cells (NECs) (Figure 1C). When grown in the presence of tumor-conditioned media, doppel was stably expressed in TECs in vitro (Supplemental Figure 6B). Most of the vasculatures expressed high levels of doppel in primary tumors (Figure 1D and Supplemental Figure 6C). Taken together, doppel expression increased ectopically in tumors and was specific to TECs at both the transcriptional and protein levels.

Doppel increases angiogenesis. Doppel was highly and ubiquitously expressed in the vasculature of squamous, breast, lung, and colon cancers (Supplemental Figure 7A). Interestingly, high doppel levels in TECs were associated with increased vessel volume in squa- 
A

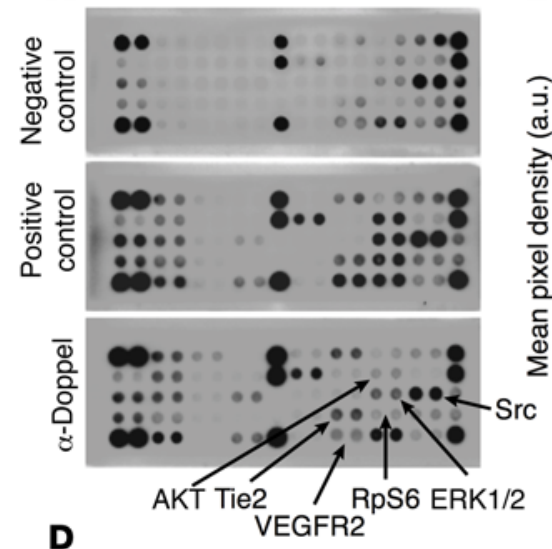

$\begin{gathered}\alpha \text {-Doppel } \\ (\mu \mathrm{g} / \mathrm{ml})\end{gathered}-\quad 0.1 \quad 1 \quad 10$ mVEGF - $++++\mathrm{kDa} E$
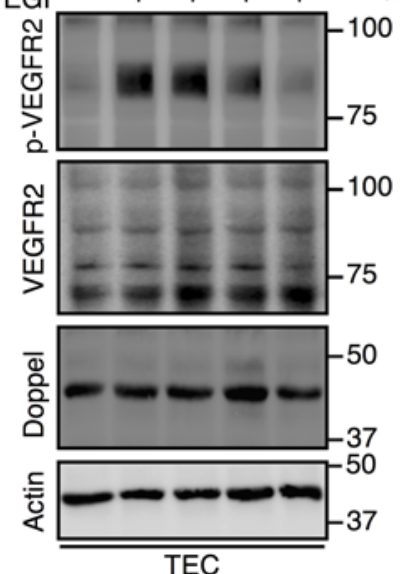

B

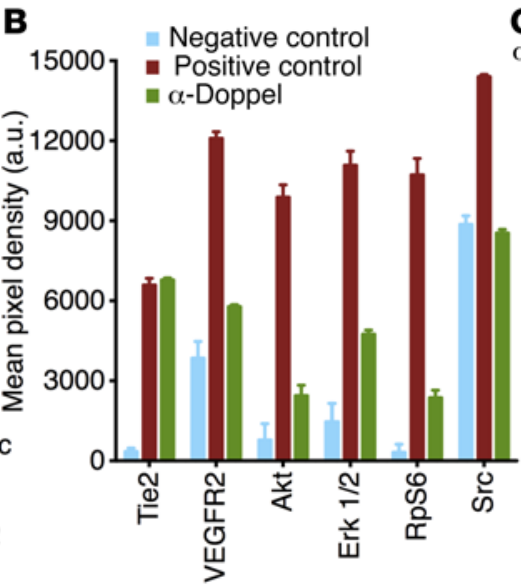

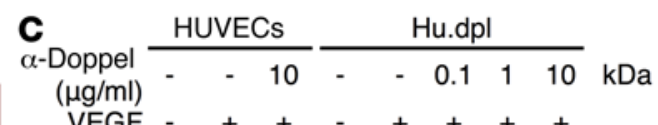

VEGF
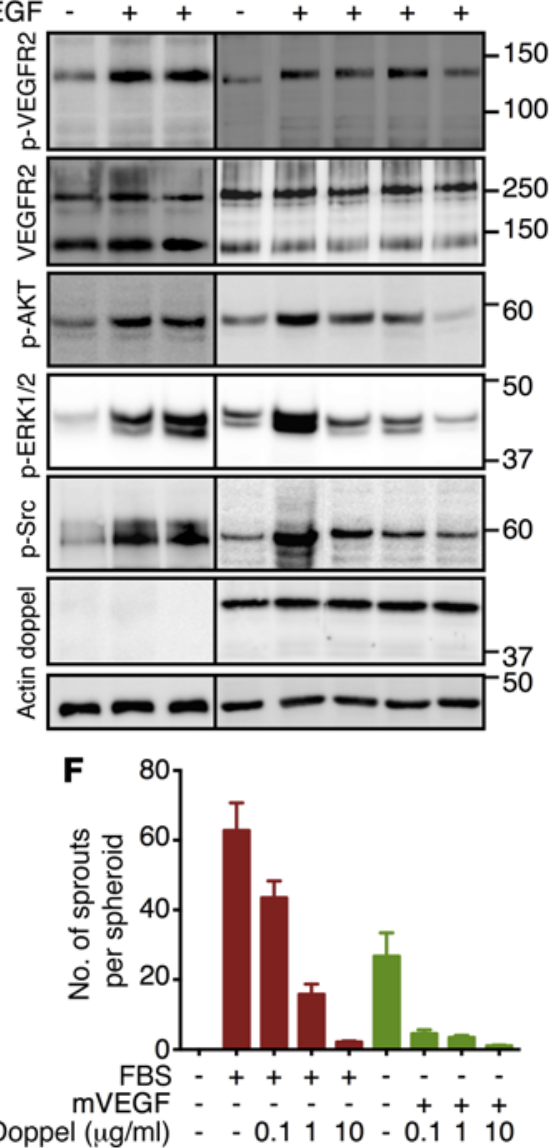

Figure 3. Doppel plays a role in VEGFR2 signaling. (A) Phosphorylated RTK (p-RTK) signaling array of Hu.dpl exposed to fasting media, complete media, and $\alpha$-doppel (30 minutes, $10 \mu \mathrm{g} / \mathrm{ml}$ ) in the presence of complete media. (B) Quantification of pixel density of p-Tie2, p-VEGFR2, p-AKT, p-ERK1/2, p-RpS6, and p-Src. See also Supplemental Figure 10. (C) Immunoblots of p-VEGFR2, p-AKT, p-ERK1/2, p-Src, and total VEGFR2, doppel, and actin in HUVECs and Hu.dpl cells treated with different concentrations of $\alpha$-doppel in the presence of VEGF $\mathrm{F}_{165}(100 \mathrm{ng} / \mathrm{ml})$. Cells were pretreated with $\alpha$-doppel for 30 minutes, and then VEGF $_{165}$ was added for 5 minutes. (D) Immunoblots of p-VEGFR2, total VEGFR2, total doppel, and actin in TECs treated with different concentrations of $\alpha$-doppel in the presence of mVEGF $(100 \mathrm{ng} / \mathrm{ml})$. Dose-dependent inhibition $(\mathbf{E})$ and total number of TEC sprouts $(\mathbf{F})$ by $\alpha$-doppel stimulated with either $10 \%$ FBS or mVEGF (100 ng/ml). Scale bar: $100 \mu \mathrm{m}$. Each experiment was repeated 3 times.

mous, breast, lung, and colon cancers (Figure 2, A and B). Tumors with increased doppel expression, determined by flow cytometry, had greater vessel volumes (Supplemental Figure 7, B and C).

On the basis of this observation, we hypothesized that doppel regulates angiogenesis in tumors. To test this hypothesis, we generated a gain-of-function EC model to express doppel in ECs. Luciferase-expressing HUVECs $\left(\mathrm{Hu}^{+ \text {tuc }}\right)$ were stably transfected with a human doppel cDNA construct $\left(\mathrm{Hu}^{\text {+luctdpl })}\right.$. The transfection efficiency was $67.8 \% \pm 8.2 \%$ before sorting, and the reanalysis of sorted $\mathrm{Hu}^{+ \text {tuctdpl }}$ cells showed $98.3 \% \pm 2.3 \%$ purity (Supplemental Figure 8A). More than $95 \%$ of transduced $\mathrm{Hu}^{+ \text {tuctdpl }}$ cells stably expressed doppel for at least 30 days (Supplemental Figure 8B). Exogenous overexpression of doppel in $\mathrm{Hu}^{\text {+luctdpl }}$ cells was observed, but there was little or no expression in nontransfected $\mathrm{Hu}^{\text {thuc }}$ cells (Supplemental Figure 8, C-F). Previous studies have established the feasibility of using primary ECs for engineering functional blood vessels in vivo (17-21). In the present study, we used a spheroid-based EC transplantation technique. ECs, inoculated in vivo in a matrix, closely mimicked the sprouting of angiogenesis $(22,23)$. The principle of this technique is that EC spheroidal aggregates are stable, induce less apoptosis than do single suspended ECs, and become highly responsive to the activities of survival factors (24). Genetically modified EC spheroids, implanted in Matrigel-fibrin gel, produced functional and durable vasculatures. Similarly, both $\mathrm{Hu}^{\text {+luc }}$ and $\mathrm{Hu}^{+ \text {tuctdpl }}$ spheroids were used to generate phenotypic perfused angiogenic vessels in vivo and to track doppel expression along with the progression of vessel formation. The growing blood vessels within their Matrigel-fibrin gel (plug) formed anastomoses with mouse vasculature that were partly covered by host-derived mural cells (Supplemental Figure 9). $\mathrm{Hu}^{\text {+luc }}$ and $\mathrm{Hu}^{\text {+luctdpl }}$ spheroids evenly vascularized within their plug and formed a vascular network (Figure 2C). The extent of vessel formation, monitored by in vivo and ex vivo bioluminescence imaging, was $8.2 \pm 0.4$ times greater in the $\mathrm{Hu}^{\text {+luctdpl }}$ plug than in the $\mathrm{Hu}^{\text {tuc }}$ plug $(P<0.001$; Figure 2, D and $\mathrm{E})$. The hemoglobin content, an indicator of perfused vessel formation, was also 8.0 
A

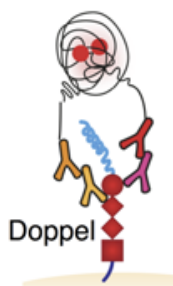

Red dots; Doppel PLA, Green; Phalloidin, Blue; Nucleus

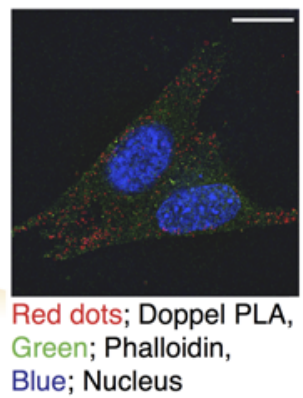

B

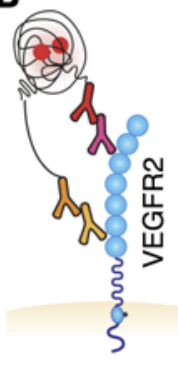

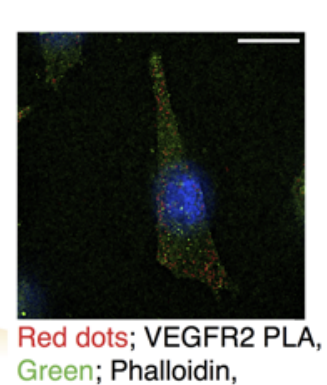
Blue; Nucleus
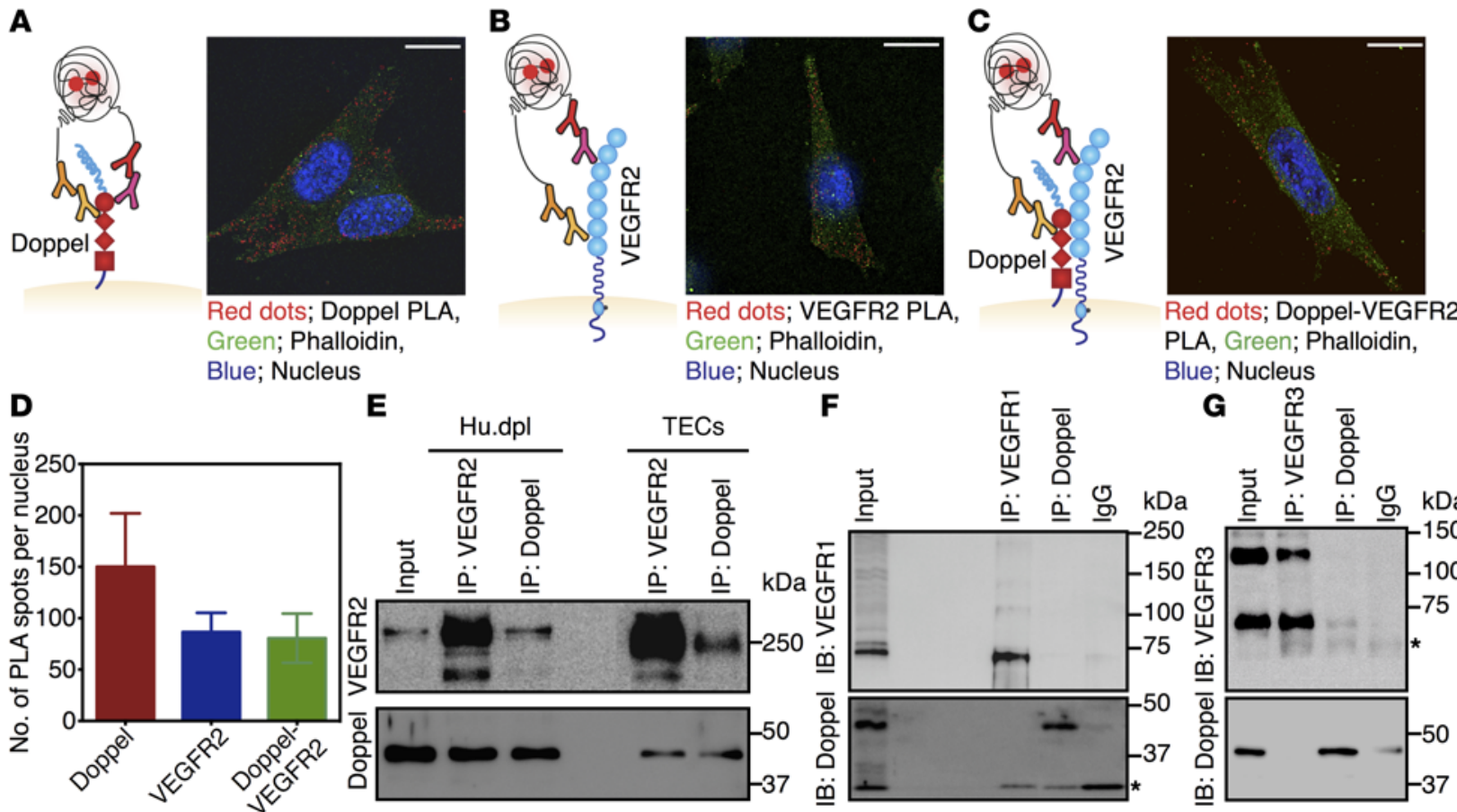

PLA, Green; Phalloidin, Blue; Nucleus

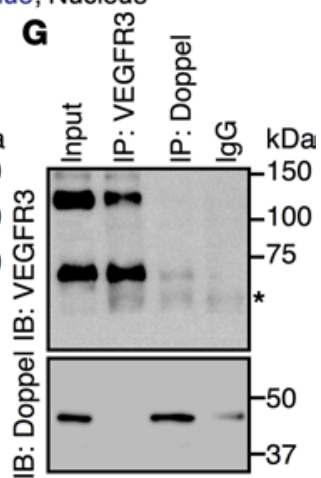

Figure 4. Doppel interacts with VEGFR2 on TECs. Representative images of the PLA of doppel (A), VEGFR2 (B), and doppel-VEGFR2 interactions (C) identified on TECs. PLA signals are shown with red dots, cytoskeletal staining (FITC-phalloidin) is shown in green, and nuclear staining (DAPI) is shown in blue. Scale bars: $5 \mu \mathrm{m}$ (A-C). (D) Quantification of doppel, VEGFR2, and doppel-VEGFR2 heterodimer PLA signals in TECs. Co-IP followed by immunoblotting (IB) of VEGFR2 and doppel in Hu.dpl cells and TECs (E), immunoblot of VEGFR1 and doppel (F), and immunoblot of VEGFR3 and doppel (G) in Hu.dpl cells. Input: whole-cell lysates. Single asterisk indicates a light or heavy chain. Each experiment was repeated 3-5 times.

\pm 0.3 times greater in the $\mathrm{Hu}^{\text {tluctdpl }}$ plug than in the $\mathrm{Hu}^{\text {+luc }}$ plug $(P<0.001$; Figure $2 \mathrm{~F})$. Whole-mount staining and confocal 3D imaging also revealed a dense and highly vascular network in the $\mathrm{Hu}^{\text {+luctdpl }}$ plug compared with that seen in the $\mathrm{Hu}^{\text {+luc }}$ plug (Figure $2 \mathrm{G})$. This phenomenon was also observed by IHC using human CD34 (hCD34) staining (Figure 2H). The angiogenic blood vessels of the $\mathrm{Hu}^{+ \text {tuctdpl }}$ plug showed pronounced doppel expression, but the $\mathrm{Hu}^{\text {+luc }}$ plug (Figure 2I) showed little or none. In the $\mathrm{Hu}^{\text {+luctdpl }}$ plug, the hCD34-positive vascular network grew $176 \pm 21$ vessels per $\mathrm{mm}^{2}$, and the doppel-positive vascular network grew $196 \pm 24$ vessels per $\mathrm{mm}^{2}$ (Figure 2J). The overall hCD34-positive vascular network was $5.4 \pm 0.6$ times greater in the $\mathrm{Hu}^{\text {+luctdpl }}$ plug than in the $\mathrm{Hu}^{\text {+luc }}$ plug $(P<0.001)$. These findings indicate that doppel expression is strongly associated with angiogenesis and that doppel could be targeted for the treatment of tumoral angiogenesis.

Doppel blocking inhibits angiogenic signaling. The mechanism of how doppel regulates angiogenesis is unknown. To determine the role of doppel expression in known angiogenic circuits, we studied the molecular basis of doppel functions and its interactions with angiogenesis. We screened the phosphorylation status of a spectrum of receptor tyrosine kinases (RTKs) and associated signaling cascades by stimulating these RTKs with complete growth media supplemented with FBS (a rich source of growth factors). HUVECs and Hu.dpl cells were stimulated and treated with $\alpha$-doppel. The cells showed a similar phosphorylation pattern when stimulated. However, VEGFR2 phosphorylation was greater in Hu.dpl cells than in HUVECs (Supplemental Figure 10). Of the 28 RTKs, phosphorylation of VEGFR2 and its associ- ated signaling hubs was substantially reduced in Hu.dpl cells upon blockage of doppel. However, no such changes were observed in HUVECs when treated with $\alpha$-doppel (Supplemental Figure 10 and Figure 3A). In addition, $\alpha$-doppel decreased the phosphorylation of AKT (Ser473), ERK1/2, RpS6, Src, and VEGFR2, but not Tie2, which suggests blocking of the VEGF-signaling pathway (Figure 3, A and B). $\alpha$-Doppel also suppressed VEGF-A-induced VEGFR2, AKT, ERK1/2, and Src phosphorylation in Hu.dpl cells in a dose-dependent manner, but this was not observed in HUVECs (Figure 3C). We also found that $\alpha$-doppel prevented mouse VEGFinduced (mVEGF-induced) VEGFR2 phosphorylation in TECs in a dose-dependent manner (Figure 3D). We then analyzed the contribution of doppel to the angiogenic functions of TECs. Doppel blocking abrogated, dose dependently, both FBS- and mVEGFinduced sprouting of TEC spheroids (Figure 3, E and F). These data suggest that doppel regulates VEGFR2 signaling in ECs.

Doppel constitutively interacts with VEGFR2. Mechanistic studies suggest that doppel complexes with VEGFR2 via a physical interaction. A proximity ligation assay (PLA) demonstrated constitutive interactions between doppel and VEGFR2 in both TECs (Figure 4, A-D, and Supplemental Figure 11, A-C) and Hu.dpl cells (Supplemental Figure 11E). The PLA signal density for doppel and VEGFR2 interactions was similar to that observed for VEGFR2 alone (Figure 4D and Supplemental Figure 11D). Co-IP experiments using isolated TECs or Hu.dpl cells also showed physical interactions between doppel and VEGFR2 (Figure 4E), but showed no interactions between doppel and VEGFR1 or doppel and VEGFR3 (Figure 4, F and G). IP of doppel by VEGFR2 Ab and VEGFR2 by 
A
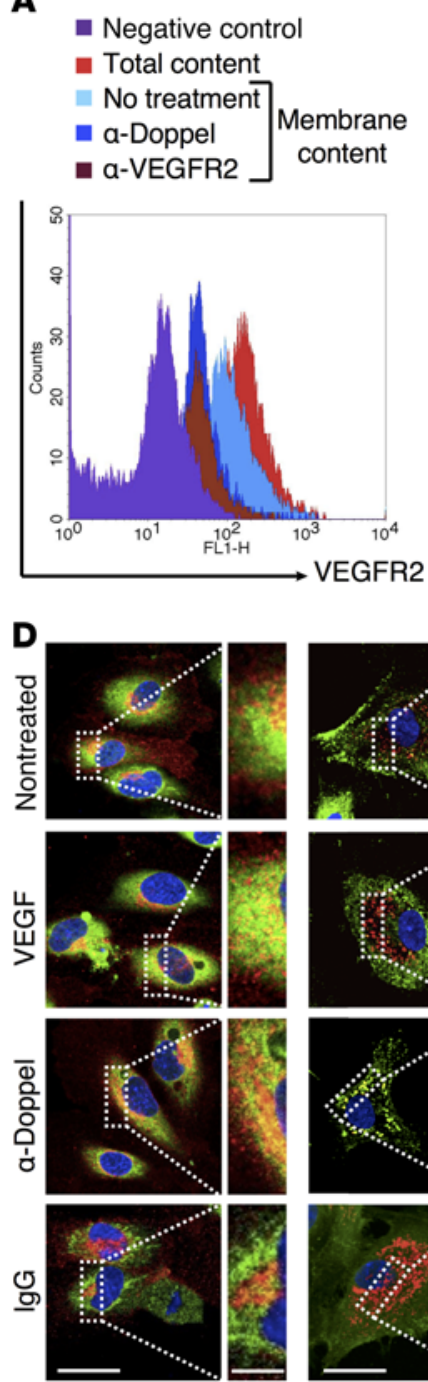

Red; VEGFR2, Green; EEA1, Blue; nucleus

G
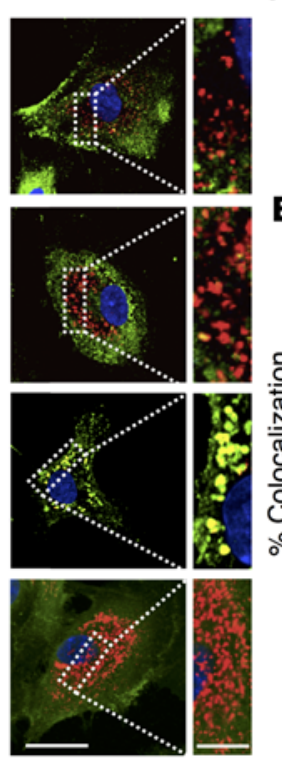

Green; VEGFR2,

Red; LAMP1,

Blue; nucleus

$\alpha$-Doppel
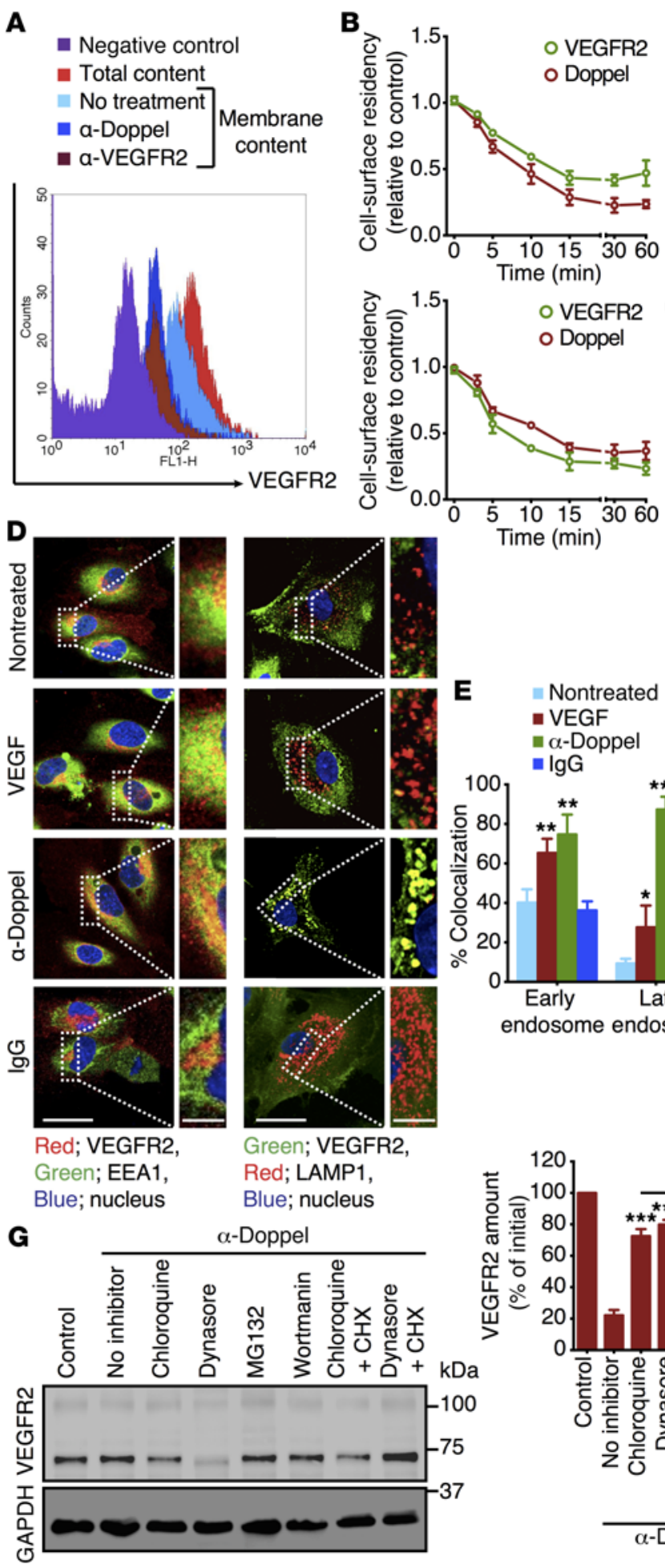

E $\quad$ Nontreated - VEGF

$\alpha$-Doppel
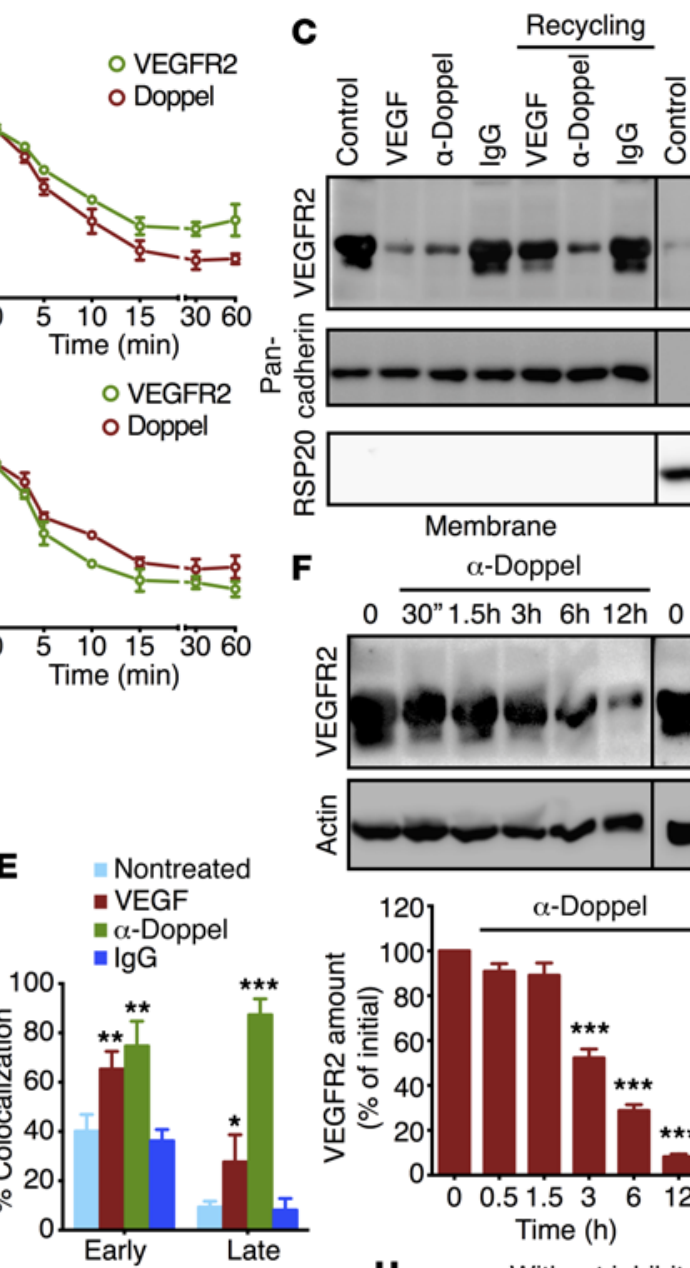

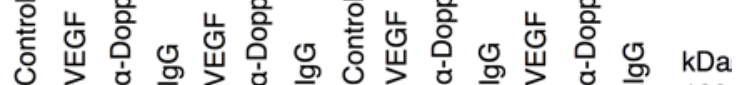

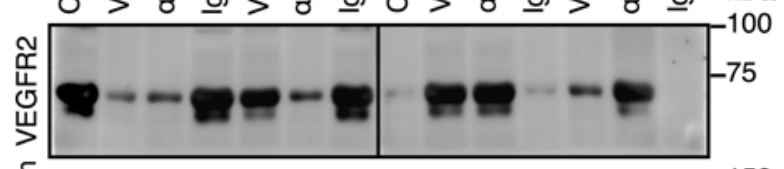

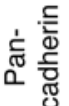
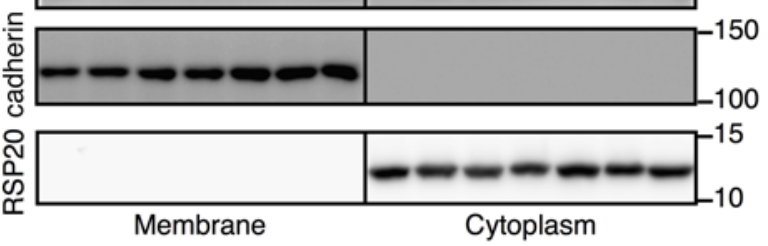

$\mathbf{F}$
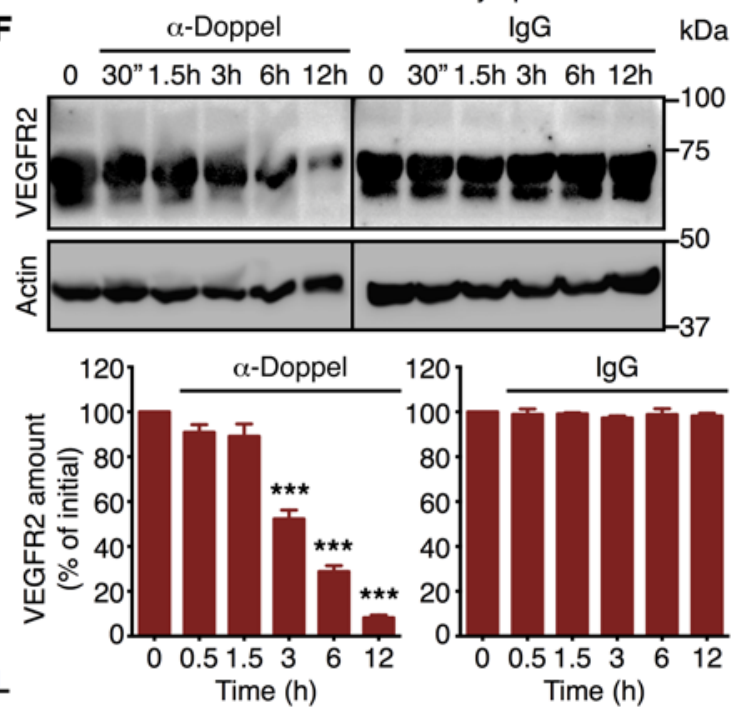
endosome endosome

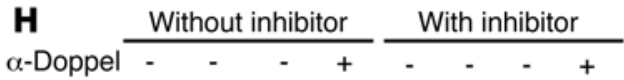
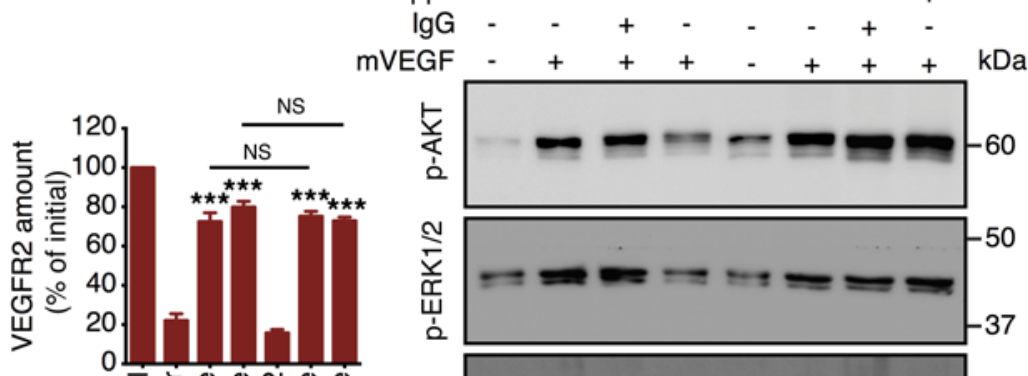

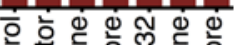

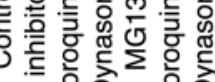

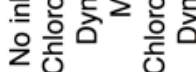

$\overline{+\mathrm{CHX}}$

$\alpha$-Doppel

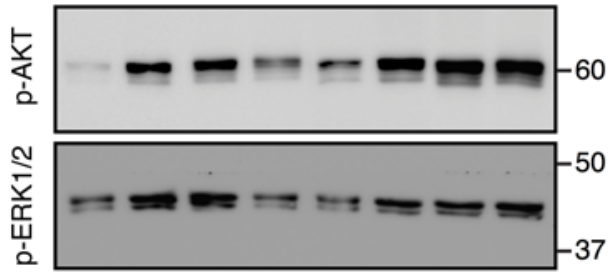

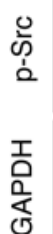

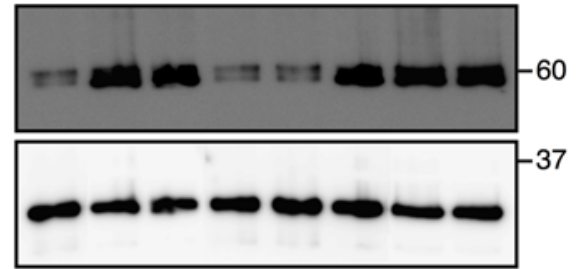


Figure 5. Doppel inhibition spatially regulates the VEGFR2 internalization process. (A) Flow cytometric analysis of VEGFR2 in permeabilized versus nonpermeabilized TECs following incubation with $\alpha$-doppel and $\alpha$-VEGFR2. VEGFR2 was internalized as a result of doppel blocking. (B) VEGFR2 and doppel internalization kinetics rate following incubation with $\alpha$-doppel (upper panel; $10 \mu \mathrm{g} / \mathrm{ml}$ ) and $\alpha$-VEGFR2 (lower panel; $10 \mu \mathrm{g} / \mathrm{ml}$ ). (C) Biochemical detection of a membrane and intracellular pool of VEGFR2 in unstimulated TECs and VEGF- (5 minutes; $100 \mathrm{ng} / \mathrm{ml}), \alpha$-doppel- (30 min; $10 \mu \mathrm{g} /$ $\mathrm{ml}$ ), and control IgG-stimulated (30 minutes; $10 \mu \mathrm{g} / \mathrm{ml}$ ) TECs. Pan-cadherin and RSP20 were used for membrane and cytoplasmic markers, respectively. (D) IF staining of TECs for VEGFR2 (red) or EEA1 (green) and VEGFR2 (green) or LAMP1 (red) following incubation with VEGF (5 min; $100 \mathrm{ng} / \mathrm{ml}$ ), $\alpha$-doppel (30 $\mathrm{min} ; 10 \mu \mathrm{g} / \mathrm{ml}$ ), and control IgG (30 $\mathrm{min} ; 10 \mu \mathrm{g} / \mathrm{ml}$ ) and (E) quantification of the colocalized fraction of fluorescence signal. Nuclei were stained with DAPI (blue). Scale bar: $10 \mu \mathrm{m}$. Panels on the right are magnified images of the outlined portion of each image (scale bar: $5 \mu \mathrm{m})$. ${ }^{* *} P<$ $0.01,{ }^{* *} P<0.01$, and ${ }^{* *} P<0.001$ versus nontreated cells, Student's $t$ test. (F) Total VEGFR2 in TECs by Western blot analysis following incubation with $\alpha$-doppel $(10 \mu \mathrm{g} / \mathrm{ml})$ and control lgG $(10 \mu \mathrm{g} / \mathrm{ml})$ at different time points. ${ }^{*} P<0.01$ and ${ }^{* * *} P<0.001$ versus initial (zero), Student's $t$ test. (G) VEGFR2 degradation rate following incubation with $\alpha$-doppel (12 hours, $10 \mu \mathrm{g} / \mathrm{ml}$ ) in the absence or presence of different endocytosis and protein translation inhibitors. ${ }^{* *} P<0.001$ versus no inhibitor, Mann-Whitney $U$ test. $(\mathbf{H}) I$ mmunoblot showing that VEGF $\left(100 \mathrm{ng} / \mathrm{ml}_{165}\right)$ stimulated the phosphorylation of AKT, ERK1/2, Src, and total GAPDH in cells when treated with $\alpha$-doppel $(10 \mu \mathrm{g} / \mathrm{ml})$ or control lgC $(10 \mu \mathrm{g} / \mathrm{ml})$ in the presence or absence of the endocytosis inhibitor dynasore. Each experiment was repeated 3 times. CHX, cycloheximide.

doppel Ab confirmed their association with each other. When stimulated with PLGF or VEGF-C, doppel blocking had no effect on the phosphorylation of VEGFR1 or VEGFR3 (Supplemental Figure 12, A and B), respectively. Doppel blocking also suppressed VEGF-Cinduced VEGFR2 phosphorylation (Supplemental Figure 12C). These results point to the fact that the doppel-VEGFR2 interaction was not an artifact caused by an overexpression in TECs or in transfected Hu.dpl cells, but the interaction was rather direct.

Spatial regulation of VEGFR 2 by blocking doppel. The mechanism for the attenuation of VEGFR2 signaling includes internalization, degradation, and dephosphorylation by specific tyrosine phosphatases (25-27). Since doppel and VEGFR2 colocalize and share the common microdomains (Supplemental Figure 13), we hypothesized that they internalize together. Indeed, doppel and VEGFR2 internalized simultaneously after incubation with $\alpha$-doppel and $\alpha$-VEGFR2 (Figure 5, A and B). In TECs, $\alpha$-doppel-mediated VEGFR2 internalization was kinetically similar to $\alpha$-VEGFR2mediated internalization. The number of internalized receptors equaled the number of receptors that were endocytosed, recycled, or degraded. Doppel-induced VEGFR2 internalization was distinctly different from that triggered by its ligand VEGF (Figure 5C). $\alpha$-Doppel and VEGF stimulation depleted membrane VEGFR2, but did not affect the intracellular pool of the receptor. When internalized by VEGF, VEGFR2 recycled back to the membrane; however, the receptor did not return to the membrane when the internalization was induced by $\alpha$-doppel. Following incubation with $\alpha$-doppel, VEGFR2 was highly internalized and colocalized with EEA1-positive endosomes $(P<0.01$ vs. control and $P<0.001$ vs. VEGF) and LAMP1-positive lysosomes $(P<0.001$ vs. control and $P<0.001$ vs. VEGF) (Figure 5, D and E). No such internalization was observed when control IgG was incubated with VEGFR2. Similar differences in the VEGFR2 turnover rate were also observed in TECs treated with $\alpha$-doppel, but not in TECs treated with control IgG (Figure 5F). The steady-state level of VEGFR2 was maintained up to 1.5 hours with treatment, and its degradation was significant after 3 hours of $\alpha$-doppel treatment. This implies that $\alpha$-doppel initially removes VEGFR2 from the cell surface, which allows VEGFR2 to accumulate in the cell's lysosomal compartments and later induce degradation. As shown in Figure 3, the amount of total VEGFR2 was the same, although the level of phosphorylation was different after 30 minutes of $\alpha$-doppel treatment. Thus, we conclude that the reduced phosphorylation of VEGFR2 was due to its induced internalization from the cell surface following doppel inhibition.
On the basis of previous reports that VEGFR2 can be degraded through proteasome- as well as lysosome-dependent pathways (28-30), we treated the cells with chloroquine (lysosomal inhibitor), dynasore (cell-permeable inhibitor of dynamin and endocytosis), MG132 (proteasome inhibitor), and wortmannin. The rate of VEGFR2 degradation significantly decreased when $\alpha$-doppel was incubated along with dynasore and chloroquine, suggesting a dynamin-mediated endocytosis and lysosome-dependent receptor degradation pathway (Figure $5 \mathrm{G}$ ). This upregulation of VEGFR2 was not due to protein translation, because no significant changes in VEGFR2 were observed when dynasore and chloroquine were cotreated with cycloheximide, a protein translation inhibitor, in the presence of $\alpha$-doppel (Figure 5G). Additionally, the ability of $\alpha$-doppel to attenuate the VEGF-signaling cascades was also investigated in the presence or absence of dynasore. $\alpha$-Doppel prevented the phosphorylation of AKT, ERK1/2, and Src in the absence of dynasore, but not in the presence of dynasore (Figure 5H). This implied that doppel inhibition in the signaling cascades was due to reduced membrane VEGFR2, although it was not a direct effect. These data, on the whole, indicate a unique spatial regulation of VEGFR2 endocytosis that occurs due to binding with doppel. Doppel inhibition reduced the membrane residency of VEGFR2, which might have made VEGF less responsive to VEGFR2. Moreover, the ability of doppel to regulate the sensitivity of TECs to proangiogenic signals suggests that doppel could be a therapeutic target in tumoral angiogenesis.

Doppel and heparin crosstalk. Previous studies suggest that heparin sulfates are involved in the pathogenesis of prion diseases and that prion proteins interact with heparin (11). Given these findings, we speculated that heparin also binds with doppel, a prion-like protein. Low-molecular-weight heparin (LMWH) binds with human PrP with a $K_{D}$ of $0.12 \mu \mathrm{M}$ (Supplemental Figure 14A). However, the $K_{D}$ for LMWH-human doppel binding was $0.43 \mu \mathrm{M}$, an approximately 3.5 -fold reduced affinity than that for PrP. To study the interactions between doppel and LMWH, we developed doppel-knockdown TECs (TEC ${ }^{-/ \text {-dpl }}$ ) by transducing shRNA with a lentiviral vector. LMWH bound with $\mathrm{TEC}^{-/-\mathrm{dpl}}$ at a markedly reduced level (Supplemental Figure 14B). LMWH nonspecifically bound to the surface of $\mathrm{TEC}^{-/ \text {-dpl }}$ cells, but colocalized specifically with doppel on the membranes of TECs. We validated the vessel-homing ability of LMWH using TEC spheroids in an in vivo angiogenesis assay, as described previously $(22,23)$. For this, Cy5.5 was chemically conjugated to 

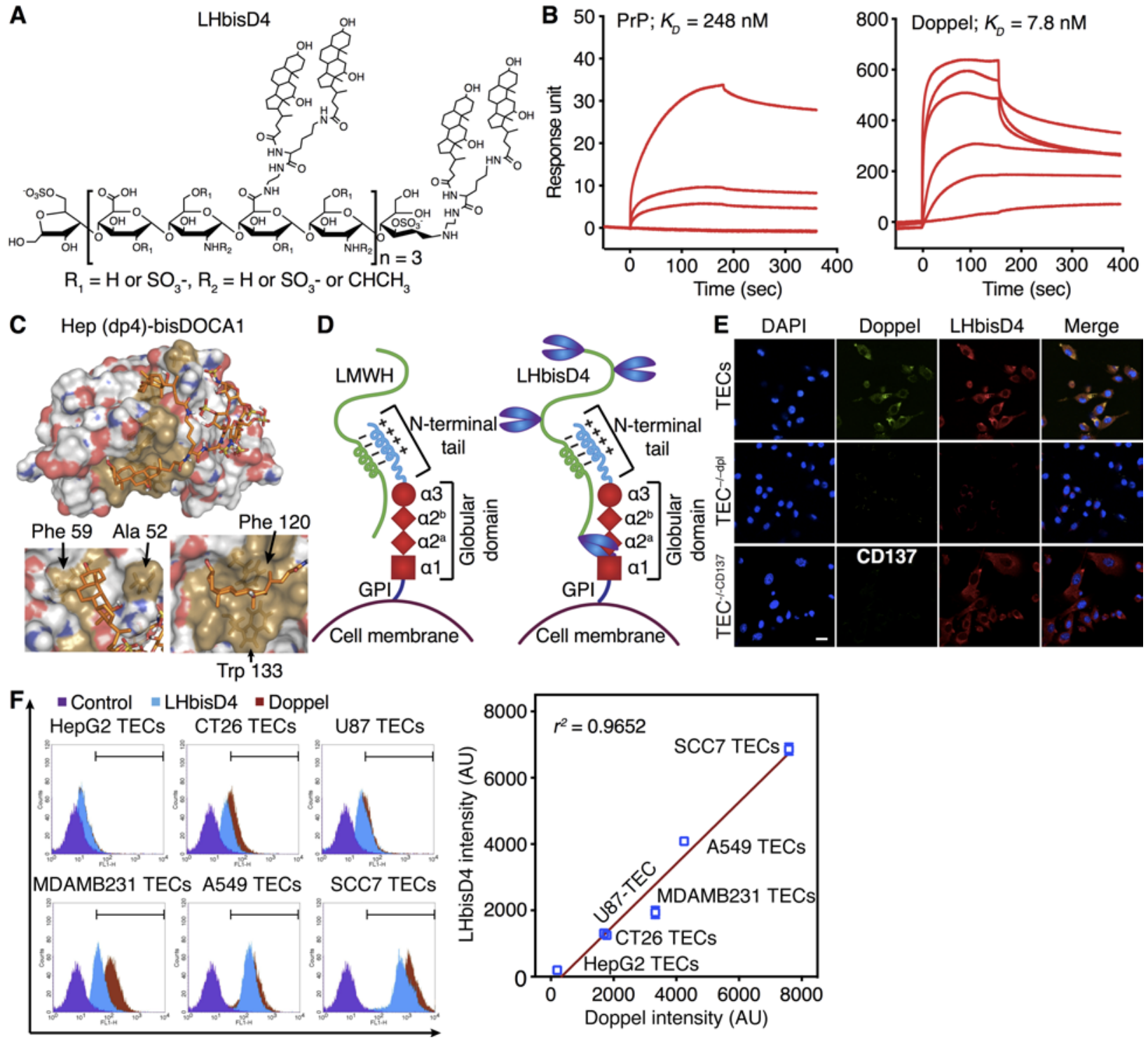

Figure 6. Heparin and its conjugate LHbisD4 can target doppel on TECs. (A) Structure of the LMWH-doxycholic acid conjugate LHbisD4, in which 4 molecules of dimeric deoxycholic acid were conjugated to 1 molecule of LMWH. (B) Surface plasmon resonance (SPR) analysis of PrP-LHbisD4 (left) and doppel-LHbisD4 (right). The $K_{D}$ was calculated from the response curves (3 experiments). (C) Globular domain structure (in light brown) of doppel in complexation with LHbisD4 fragments (upper). Detailed view of the LHbisD4 fragment-binding sites (lower panels). Residues interacting with the LHbisD4 fragments are shown as orange sticks and are labeled. (D) Proposed mechanism of LMWH and LHbisD4 binding with doppel. The basic residues of the flexible N-terminal end of doppel facilitate an interaction with the negatively charged LMWH. The conjugation of deoxycholic acids allows additional hydrophobic binding with the globular $\alpha-2 a$ and $\alpha-2 b$ helical secondary structure of doppel. The proposed site of direct interaction is near the glycosylation sites; therefore, it may not be as accessible as suggested by the modeling or the studies with recombinant doppel. (E) LHbisD4 binding with TECs, doppel-depleted TECs (TEC $\left.{ }^{-/- \text {dpl }}\right)$, and CD137-knockdown TECs (TEC $\left.{ }^{-/-C D 137}\right)$. Scale bar: $20 \mu \mathrm{m}$ ( $n=3$ experiments). (F) Correlation between doppel expression and LHbisD4 binding in isolated TECs of different cancerous cell lines was determined by flow cytometry ( $n=3$ experiments). LHbisD4 bound with different TECs, depending on doppel expression.

LMWH. This dye conjugation did not change the binding affinity of LMWH for PrP or for doppel; the $K_{D}$ values for the Cy5.5LMWH conjugate were $0.18 \mu \mathrm{M}$ and $0.51 \mu \mathrm{M}$ (Supplemental Table 1). Both TEC and TEC $^{-/- \text {dpl }}$ spheroids in the Matrigel plugs were grafted into the flanks of mice that had perfused vascular networks. However, the extent of vessel formation and hemoglobin content was much less in the $\mathrm{TEC}^{-/ \text {dpl }}$ plug than in the TEC plug (Supplemental Figure 14, C and D). The accumulation of Cy5.5 LMWH, injected into the tail vein, was greater in the
TEC plug than in the TEC $^{-/ \text {dpl }}$ plug (Supplemental Figure 14, $\mathrm{E}-\mathrm{H})$. The images of isolated plugs also confirmed that LMWH was distributed more in the TEC plug than in the $\mathrm{TEC}^{-/ \text {dpl }}$ plug (Supplemental Figure 14F). LMWH localized extensively in the growing tumoral blood vessels of the TEC plug, but little localization was observed in the blood vessels of the $\mathrm{TEC}^{-/ \text {dpl }}$ plug (Supplemental Figure 14G). LMWH accumulation was 2.3- \pm 0.4-fold greater in the TEC plug than in the $\mathrm{TEC}^{-/ \text {-dpl }}$ plug, when the fluorescence intensity of each plug was normalized with 

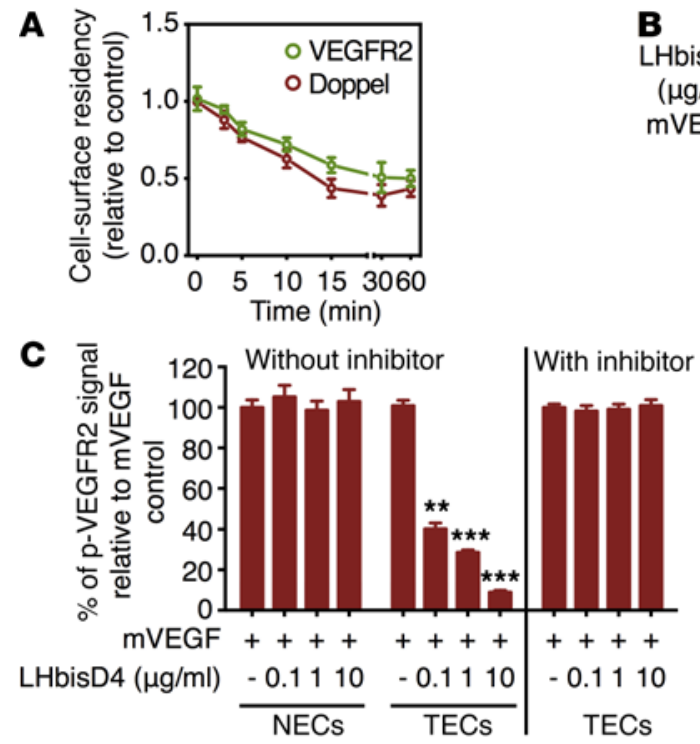

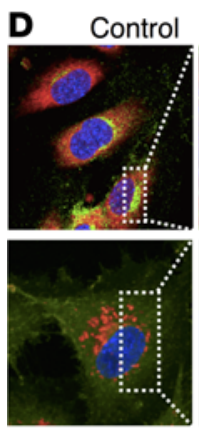

NECs

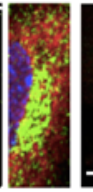

a

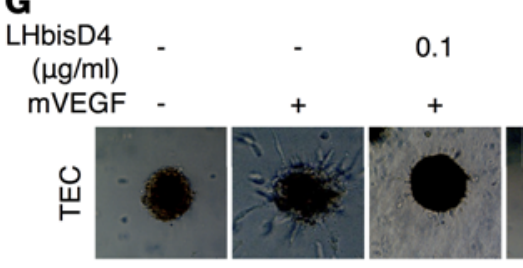

B

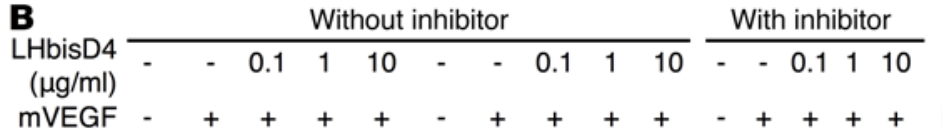
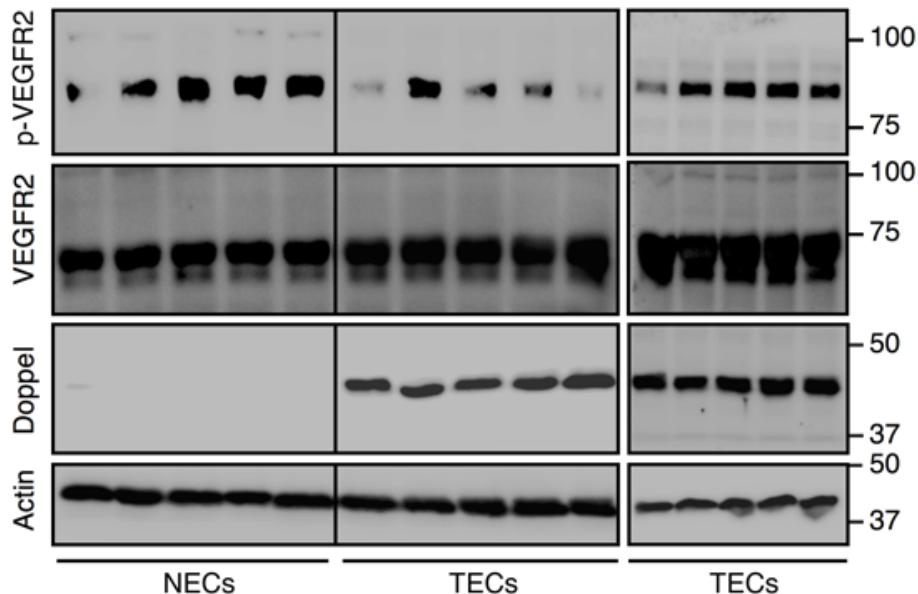

$\mathbf{F}$

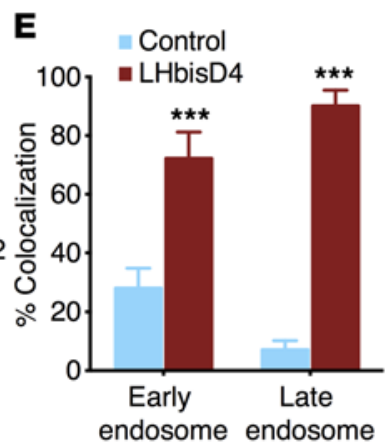

Red; VEGFR2 Green; EEA1,

Blue; nucleus
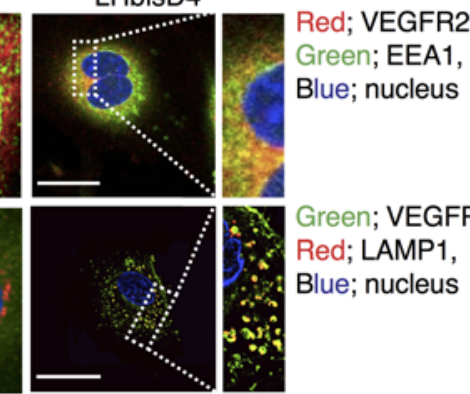

Green; VEGFR
Red; LAMP1,
Blue; nucleus
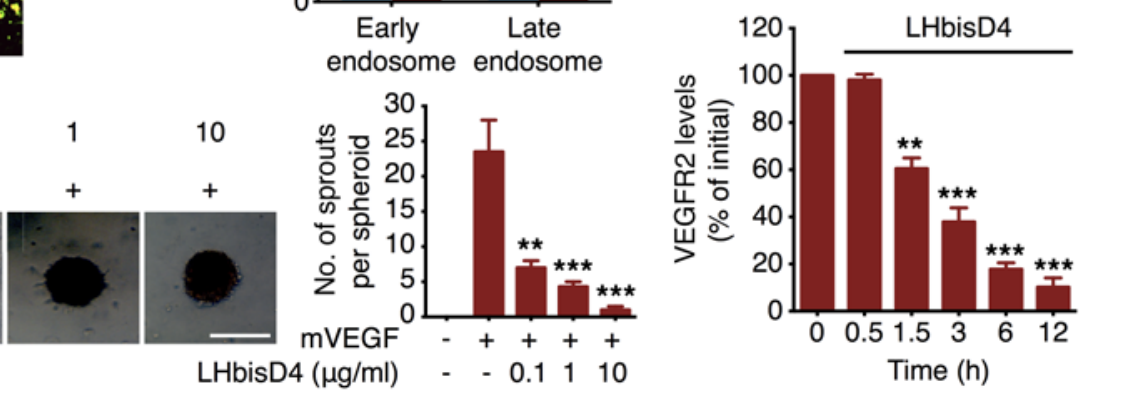

Figure 7. LHbisD4 inhibits angiogenic signaling in TECs. (A) Flow cytometric analysis of VEGFR2 in nonpermeabilized TECs following incubation with LHbisD4 (10 $\mu \mathrm{g} / \mathrm{ml})$ at different time points. (B) Immunoblot of mVEGF-stimulated (100 ng/ml) phosphorylation of VEGFR2, total VEGFR2, total doppel, and actin in NECs derived from brain and in TECs following incubation with different concentrations of LHbisD4 in the presence or absence of the endocytosis inhibitor dynasore. Dynasore was pretreated for 2 hours prior to the incubation of LHbisD4. Cells were then treated with LHbisD4 for 30 minutes and stimulated with mVEGF for 5 minutes. (C) Densitometric measurement of the p-VEGFR2 signal (normalized to VEGFR2 and actin bands) from each experiment. Results are expressed as percentages relative to the mVEGF-treated group. ${ }^{*} P<0.01$ and ${ }^{* *} P<0.001$ versus mVEGF treatment alone, Mann-Whitney $U$ test. (D) Representative images of TECs with staining for VEGFR2 (red) or EEA1 (green) and VEGFR2 (green) or LAMP1 (red) following incubation with LHbisD4 (30 min; $10 \mu \mathrm{g} / \mathrm{ml}$ ). Nuclei were stained with DAPI (blue). Scale bars: $10 \mu \mathrm{m}$. Panels on the right are magnified images of the outlined portion of each image (scale bars: $5 \mu \mathrm{m}$ ). (E) Colocalized fraction of fluorescence signal between VEGFR2 and EEA1 or LAMP1. ${ }^{* *} P<0.001$ versus control, Student's $t$ test. (F) Total VEGFR2 in TECs by Western blotting following incubation with LHbisD4 $(10 \mu g / \mathrm{ml})$ at different time points. ${ }^{* *} P<0.01$ and ${ }^{* * *} P<0.001$ versus initial (zero), Student's $t$ test. (G) TEC-sprouting assay following incubation with different concentrations of LHbisD4 in the presence or absence of mVEGF. Scale bar: $100 \mu \mathrm{m} .{ }^{* *} P<0.01$ and ${ }^{* * *} P<0.001$ versus mVEGF treatment alone, Student's $t$ test. Each experiment was performed 3 times.

their respective hemoglobin contents (Supplemental Figure $14 \mathrm{H})$. These results showed that heparin-like GAGs target the doppel-expressing tumoral endothelium. Thus, given the scaffold of heparin, we sought to develop a highly potent and selective molecule that could bind doppel efficiently.

Targeting doppel with the heparin-based conjugate LHbisD4. We designed new heparin-based compounds by conjugating heparin with DOCA. We selected LHbisD4, a chemical conjugate with a ratio of 4 dimeric DOCA molecules to 1 LMWH molecule (Supplemental Figure 15A and Figure 6A). LHbisD4 had a higher binding affinity for doppel $\left(K_{D}=7.8 \mathrm{nM}\right)$ than did LMWH or other bile acid-based conjugates (Supplemental Figure 15B and Figure 6B). The anti-FXa activity, which represents the anticoagulant activity of LHbisD4, was $0.0 \pm 0.0 \mathrm{IU} / \mathrm{mg}$ (Supplemental Table 1). Unlike LMWH, LHbisD4 showed no change in its binding affinity for the cellular prion protein (Figure 6B). 

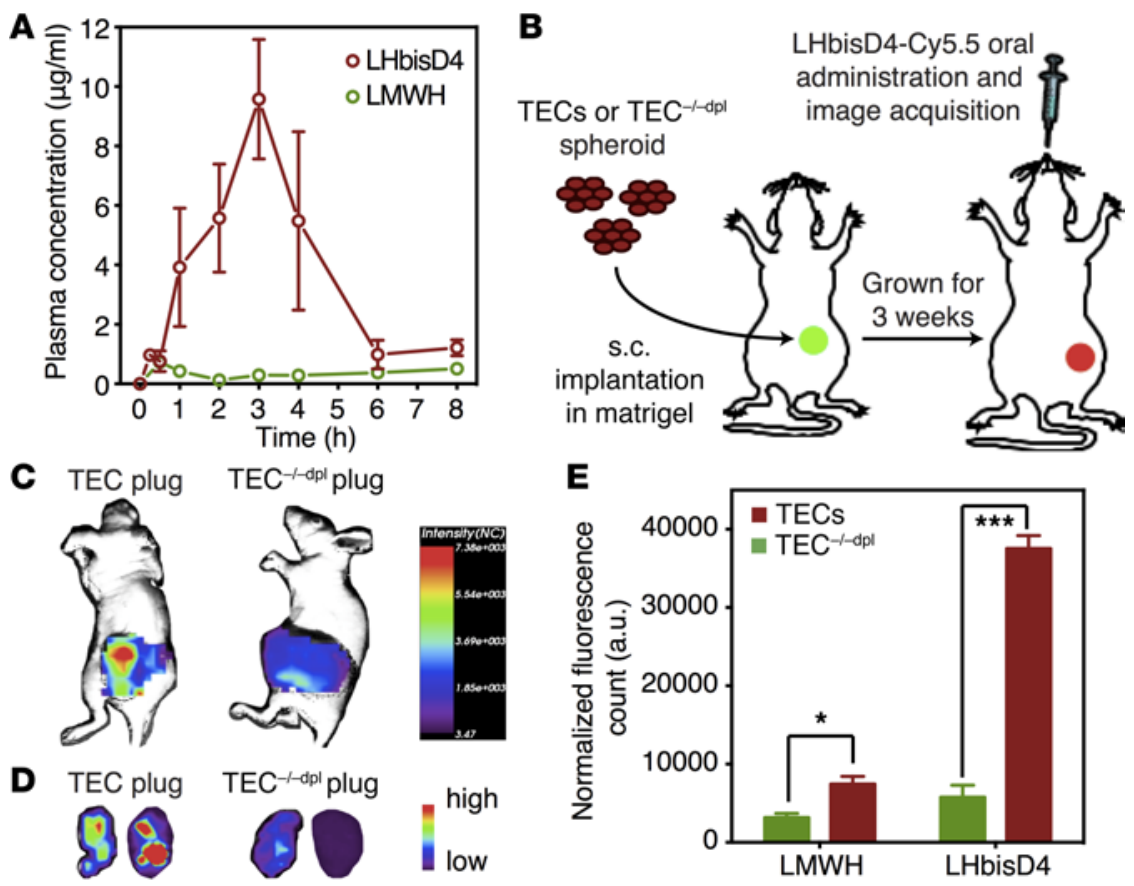

Figure 8. In vivo TEC homing ability of LHbisD4 following oral delivery. (A) Absorption of LMWH and LHbisD4 in rats after oral delivery at a dose of $10 \mathrm{mg} / \mathrm{kg}$ ( $n=4-6$ rats). (B) Experimental procedure to evaluate the ability of LHbisD4 to target doppel in vivo. Cy5.5-labeled LHbisD4 (10 mg/kg) was administered orally to female BALB/c nude mice that were s.c. implanted with SCC7-derived TECs and doppel-depleted TEC (TEC 1 -dpl) spheroids. A perfused vascular network formed within 21 days of implantation. In vivo distribution (C) and ex vivo image (D) of Cy5.5-labeled LHbisD4 in TECs and

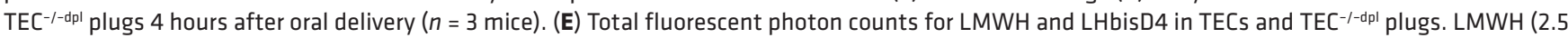
$\mathrm{mg} / \mathrm{kg}$ ) was injected i.v., and LHbisD4 $(10 \mathrm{mg} / \mathrm{kg})$ was administered orally. All values were normalized to the hemoglobin content of each plug. ${ }^{*} P<0.05$ and ${ }^{* *} P<0.001$ for TECs versus TEC ${ }^{-1-\text { dpl }}$, Student's $t$ test.

Fragment-based structural studies using computer simulation showed that the globular domain of doppel plays a critical role in binding with LHbisD4, because this domain contains hydrophobic grooves to capture large hydrophobic side chains of LHbisD4 (Figure 6, C and D). The binding of the globular domain of doppel with LHbisD4 displayed stable intermolecular interactions with a reduced energy state $(-7.6 \mathrm{kcal} / \mathrm{mol})$. The binding between the conjugated dimeric DOCA moieties of LHbisD4 and the globular $\alpha-2(\alpha-2 a$ and $\alpha-2 b)$ helical secondary structure of doppel was primarily mediated by the hydrophobic interactions of Ala 52, Phe 59, Phe 120, and Trp 133. Therefore, optimizing the structure with DOCA conjugation enhanced the binding affinity of LHbisD4 for doppel. To determine the specificity of LHbisD4 for doppel, we also evaluated the binding affinity of LHbisD4 for CD137, a known TEC marker. Tumor sections and TECs isolated from the SCC7 model showed higher expression levels of CD137 (Supplemental Figures 16, A-C). LHbisD4 binding was reduced to a greater extent in $\mathrm{TEC}^{-/ \text {-dpl }}$ than in TECs, but no change was observed in CD137-knockdown cells (Figure 6E). LHbisD4, unlike LMWH, showed no increase in its binding affinity for CD137 (Supplemental Figure 16D). LHbisD4 binding was strongly associated with doppel expression in TECs from various tumors (Figure 6F). Notably, TECs from HepG2 cells (liver cancer cells) expressed little doppel, and the binding of LHbisD4 with doppel was rather weak. These results confirmed that LHbisD4 specifically binds with doppel.
LHbisD4 targeting to doppel attenuates the angiogenic signaling of TECs. Polyanions dramatically decrease cellular PrP from cell surfaces by enhancing its rate of endocytosis; internalization of PrP is mediated by clathrin-coated pits and vesicles (31). Because doppel is a PrP, we hypothesized that the binding of doppel and heparin or heparin-based sulfated compounds induces rapid and extensive alterations in the cellular distribution of doppel. The ability of LHbisD4 to attenuate doppel-VEGFR2 interactions was also confirmed in vitro. LHbisD4 depleted doppel and VEGFR2 from TEC membranes (Figure 7A), which may lessen the responsiveness of VEGF to VEGFR2. LHbisD4 prevented mVEGFinduced VEGFR2 phosphorylation in TECs in a dose-dependent manner. However, this phenomenon was not observed when cells were treated in the presence of dynasore, a dynamin-dependent endocytosis inhibitor (Figure 7, B and C). Similar to $\alpha$-doppel, VEGFR2 was also endocytosed and colocalized with EEA1positive endosomes $(P<0.001$ vs. control) and LAMP1-positive lysosomes ( $P<0.001$ vs. control; Figure 7, D and E) upon LHbisD4 treatment, suggesting that LHbisD4 induced an internalization pathway similar to that induced by $\alpha$-doppel, which redistributed VEGFR2 in TECs. Examination of total VEGFR2 by Western blotting showed that LHbisD4 degraded and reduced the levels of this receptor (Figure 7F). LHbisD4 also dose dependently blunted mVEGF-induced sprouting of TEC spheroids (Figure 7G). These results indicate that LHbisD4 specifically inhibits the angiogenic signaling of TECs by binding with doppel. 

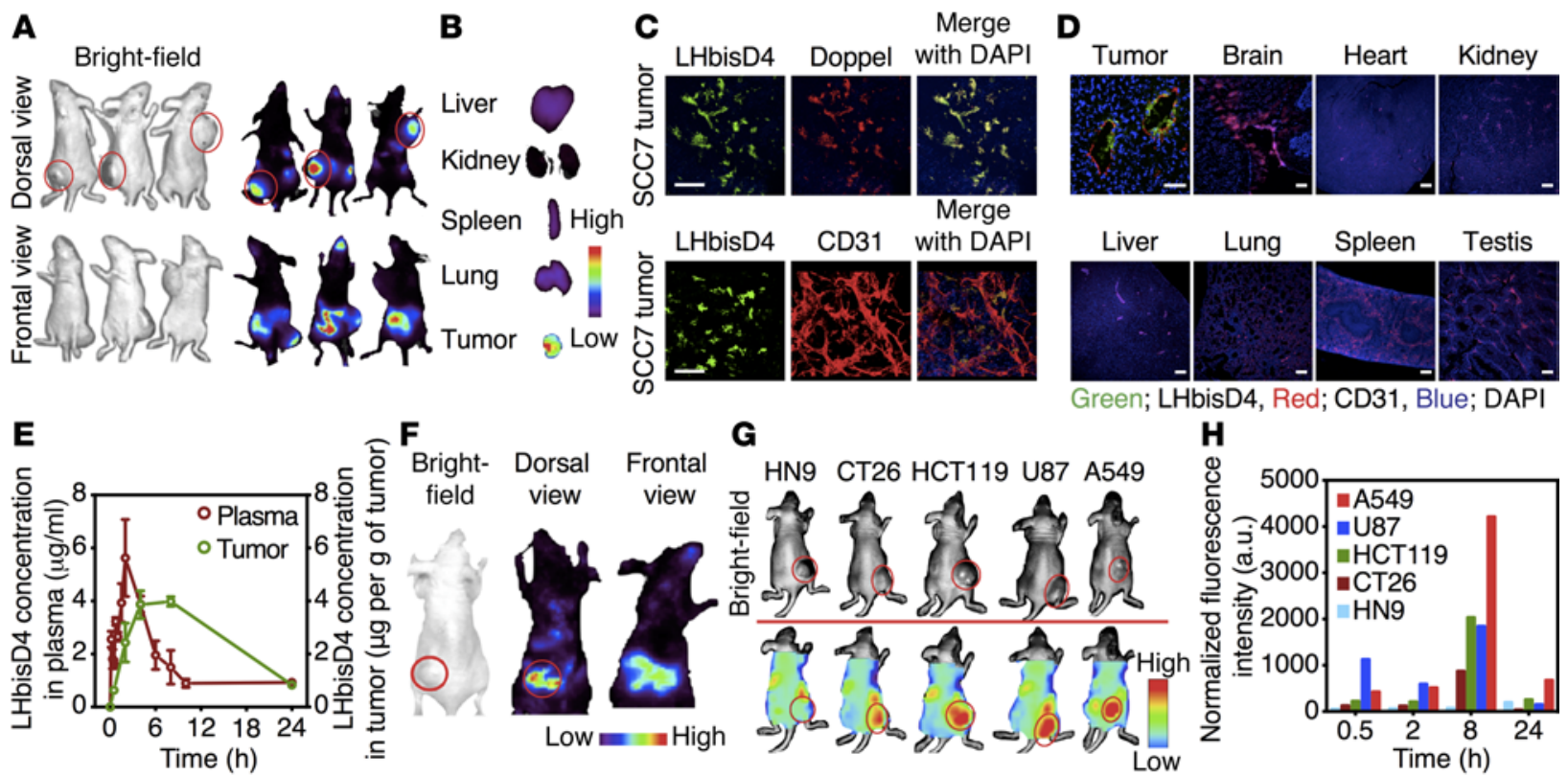

G

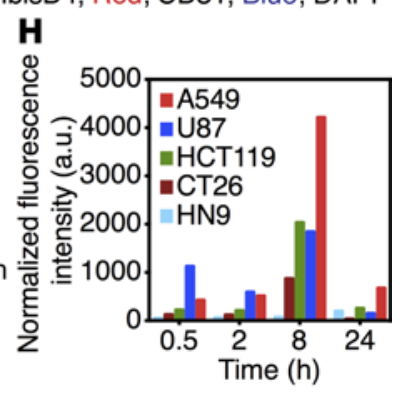

Figure 9. LHbisD4 targets doppel-expressing vasculatures with broad tumor specificity. Whole-body distribution (A) and organ accumulation (B) of Cy5.5-labeled LHbisD4 in SCC7 tumor-bearing mice 8 hours after oral administration at a dose of $10 \mathrm{mg} / \mathrm{kg}(n=3$ mice). See also Supplemental Figure 5, A and B. (C) Localization of LHbisD4 in the SCC7 tumor ( $n=3$ tumors). LHbisD4 was mainly localized in the doppel-expressing blood vessels of tumor sections. Scale bars: $20 \mu \mathrm{m}$. (D) LHbisD4 localization in vivo was assessed by IF staining of various organs following oral administration of Cy5.5-labeled LHbisD4 (10 $\mathrm{mg} / \mathrm{kg}$ ) to SCC7 tumor-bearing mice ( $n=3$ tumors). LHbisD4 is stained in green, blood vessels in red, and nuclei in blue. Scale bars: $50 \mu \mathrm{m}$ (tumor sections) and $100 \mu \mathrm{m}$ (other organs). (E) Amount of LHbisD4 in the plasma and in SCC7 tumor at different time points after oral administration at a dose of $10 \mathrm{mg} / \mathrm{kg}$ ( $n=4$ mice). (F and $\mathbf{G}$ ) Cy5.5-labeled LHbisD4 distribution in 6 different tumor models of different cancer types (breast, head and neck, colorectal, brain, and lung cancers) in mice 8 hours after oral administration at a dose of $10 \mathrm{mg} / \mathrm{kg}$ and $(\mathbf{H})$ total photon counts in the tumors at different time points.

Orally absorbed LHbisD4 attacks TECs that express doppel. Oligo-or polysaccharides cannot traverse the enterocyte layer in the small intestine, which constitutes the first step in oral availability. We previously showed that the conjugation of DOCA or oligomeric DOCA promotes oral absorption of heparin via apical sodium-dependent bile acid transporters in the intestine (32-34). LHbisD4 showed enhanced tissue permeability in vitro (Supplemental Table 1), a plasma concentration of $9.3 \pm 1.4 \mu \mathrm{g} /$ $\mathrm{ml}$, and an oral bioavailability of $23.4 \% \pm 4.6 \%$ after administration to rats (Figure 8A and Supplemental Table 2). The targeting ability of LHbisD4 was evaluated first in spheroid-based angiogenesis models using TECs and $\mathrm{TEC}^{-/ \text {dpl }}$ cells (Figure $8 \mathrm{~B}$ ). LHbisD 4 was also labeled with Cy5.5; the $K_{D}$ was similar to that of the unlabeled LHbisD 4 for both PrP and doppel (Supplemental Table 1). Orally administered Cy5.5-labeled LHbisD4 showed a higher fluorescence signal in the TEC plug than that detected in the $\mathrm{TEC}^{-/ \text {-dpl }}$ plug (TEC/TEC ${ }^{-/ \text {-dpl }}$ ratio 6.5- \pm 0.8 -fold, $P<0.001$; Figure 8, C-E). These results suggest that LHbisD4 targets the doppel-expressing endothelium in tumor tissue.

Homing efficiency of LHbisD4 to tumor vasculatures. We further assessed the vasculature-targeting ability of LHbisD4 in a tumor models. High fluorescence intensity of LHbisD4-Cy5.5 at the SCC7 tumor site was observed 1 hour after oral administration of LHbisD4-Cy5.5 to mice (Figure 9A and Supplemental Figure 17A). The fluorescence intensity at the tumor site considerably increased for 12 hours, but gradually decreased in the intestine. The uptake of LHbisD 4 by the tumor tissue was higher than uptake by normal tissues such as liver, kidney, spleen, and lung (Figure 9B and Supplemental Figure 17B). Total fluorescent photon counts in the tumor tissue were approximately 0.7- $\pm 0.2-, 4.2- \pm 0.9-, 23.4- \pm 4.7-, 5.4- \pm 0.5-$, and 2.9- $\pm 0.2-$ fold greater at $0.5,2,8,12$, and 24 hours than photon counts in the liver (Supplemental Figure 17C). In tumor tissue, LHbisD4 colocalized with doppel- and CD31-positive vasculatures (Figure 9C). LHbisD4 localized exclusively in the tumor-associated vasculatures, but not in the control tissues such as brain, heart, kidney, liver, lung, spleen, and testis (Figure 9D). Drug concentrations in the tumor tissue and in plasma were quantified by measuring the fluorescence intensity of Cy5.5-labeled LHbisD4 (Figure 9E). The plasma concentration of Cy5.5-labeled LHbisD4 reached a maximum at 2 hours after oral administration, whereas the drug concentration at the tumor site reached a maximum 4 to 8 hours after oral administration. A large amount of LHbisD4 was also accumulated in different s.c. cancer models such as colorectal cancer (CT26 and HCT119), brain cancer (U87), breast cancer (MDAMB-231), and lung cancer (A549). However, little or no accumulation was observed in the head and neck cancer (HN9), which was found to be a poorly developed, nonendothelium-based angiogenic tumor in vivo (Figure 9, F-H, and Supplemental Figure 17D). A comprehensive immunohistochemical analysis of 6 different tumors revealed that the distribution of LHbisD4 was mostly restricted to tumor vessels (Supplemental Figure 17E). Consistent with the data in Figure $3 \mathrm{~F}$, where doppel expression was approximately 3 -fold higher in 

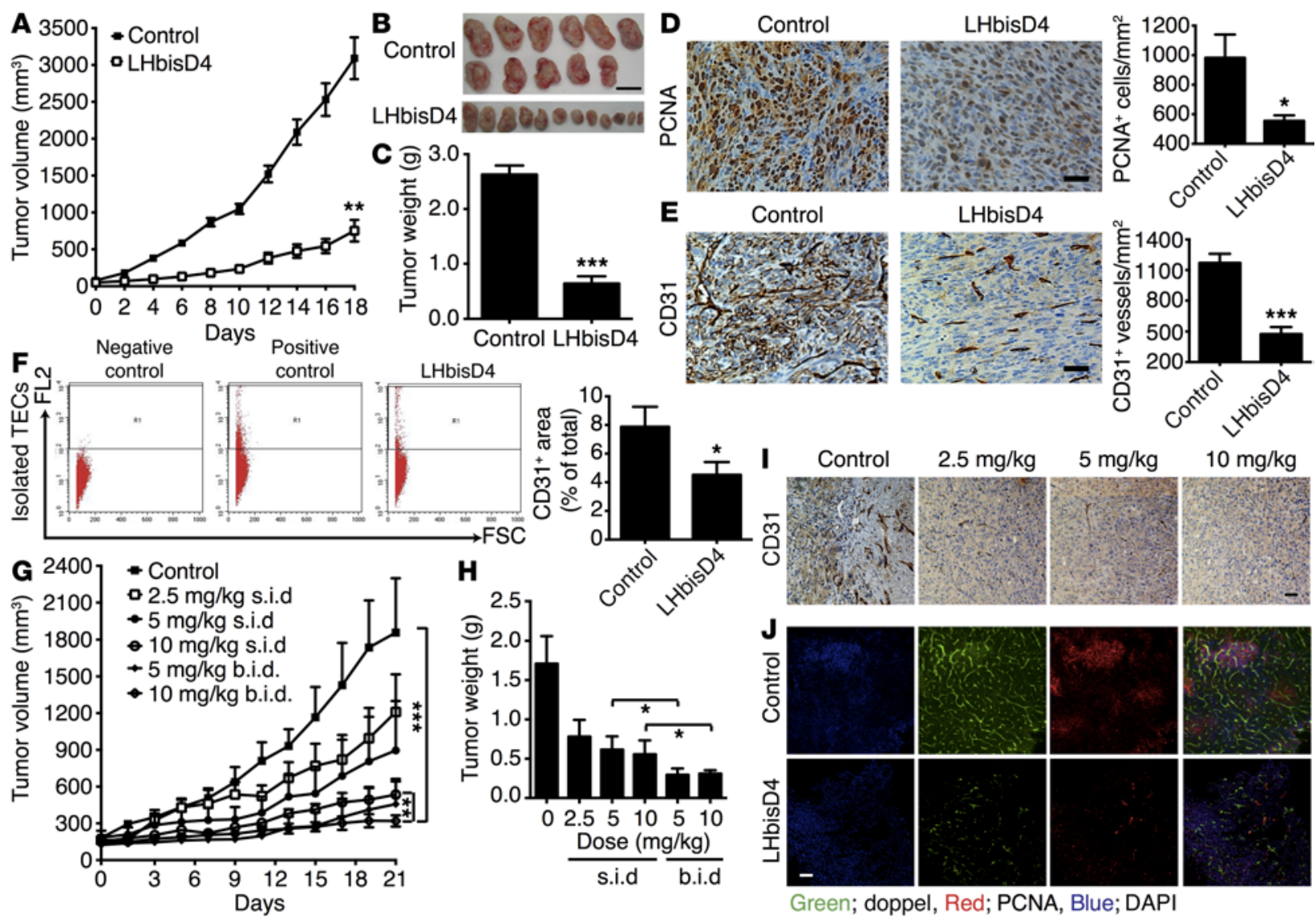

Fich 10 . Therapeutic efficacy of LHbisD4. (A) Orally administered LHbisD4, at a dose of $10 \mathrm{mg} / \mathrm{kg}$ daily, inhibited SCC7 tumor growth ( $n=11-12 \mathrm{mice}$ ). ${ }^{* *} P<0.01$ versus control, Student's $t$ test. (B) Images of isolated tumors after termination of the experiment. Scale bar: $1 \mathrm{~mm}$. (C) Tumors were excised after the study to calculate the final tumor weight. ${ }^{* *} P<0.001$ versus control. (D and E) Representative images of SCC7 tumor-bearing mouse tumor sections stained for PCNA (proliferating cells) and CD31 (blood vessels) and their staining score $\left(n=11\right.$ mice). Scale bars: $50 \mu \mathrm{m}$. ${ }^{*} P<0.05$ versus control; ${ }^{* *} P<0.001$ versus control, Student's $t$ test. (F) Total volume of isolated TECs after termination of the experiment ( $n=11$ tumor sections). ${ }^{*} P<$ 0.01 versus control, Student's $t$ test. (G) Tumor growth inhibition study of orally administered LHbisD4 in MDAMB-231 tumor at doses of 2.5, 5, and 10 $\mathrm{mg} / \mathrm{kg}$ once daily or 5 and $10 \mathrm{mg} / \mathrm{kg}$ twice daily $\left(n=5-7\right.$ mice). ${ }^{* * *} P<0.001$ between each of the groups and the control group. ${ }^{* *} P<0.01$ between the $10 \mathrm{mg} / \mathrm{kg}$ once daily and the $10 \mathrm{mg} / \mathrm{kg}$ twice daily groups, Mann-Whitney $U$ test. (H) Tumors were excised at the end of the study to calculate the final tumor weight. ${ }^{*} P<0.05$, Mann-Whitney $U$ test. (I) Tumor sections from MDAMB-231 tumor-bearing mice were stained for CD31 (blood vessels) after LHbisD4 treatment at different doses ( $n=5-7$ mice). Scale bar: $50 \mu \mathrm{m}$. (J) Dual staining of doppel (green) and PCNA (red) in a section of control and LHbisD4-treated samples. Scale bar: $50 \mu \mathrm{m}$.

TECs from A549 tumors than in those from U87 tumors, LHbisD4-Cy5.5 fluorescence intensity was also 2.5 times greater in A549 tumors than in U87 tumors. These data illustrate that the tumor-targeting ability of LHbisD4 depends on the degree of doppel expression in TECs.

Effects of LHbisD4 on tumor vasculature and growth. The antitumor efficacy of LHbisD4 was evaluated in SCC7 and MDAMB-231 tumor-grafted mouse models. When LHbisD4 (10 $\mathrm{mg} / \mathrm{kg}$ ) was orally administered once a day to mice with SCC7 xenograft tumors, both tumor volume and weight decreased significantly when compared with tumors from saline-treated mice (Figure 10, A-C). Tumor tissues from LHbisD4-treated mice showed a greater reduction in blood vessel density and proliferating tumor cells than was seen in the tumor tissues from control group mice (Figure 10, D and E). The total volume of ECs in the LHbisD4-treated and control groups was $4.5 \% \pm 0.9 \%$ and $7.9 \% \pm 1.3 \%$, respectively $(P<0.01$; Figure $10 \mathrm{~F})$. The antitumor effect of LHbisD4 was also observed in MDAMB-231 human breast carcinoma xenograft models (Figure 10, G-I). LHbisD4 inhibited tumor growth in a dose-dependent fashion when the dose was increased to $10 \mathrm{mg} / \mathrm{kg}$. The blood vessel density also decreased commensurately with the dose (Figure 10I). Moreover, LHbisD4-treated MDAMB-231 tumors had fewer doppelpositive vessels (green) and proliferating cells (red) than did the control group tumors (Figure 10J). These data demonstrate that doppel targeting selectively inhibits angiogenesis in tumors.

Together, these results indicate that spatial regulation of VEGFR2 endocytosis occurs in TECs, but not in NECs, due to doppel inhibition (Figure 11). We have shown that the binding of LHbisD4 enhances the constitutive internalization of doppel. Furthermore, we observed that doppel and VEGFR2 colocalize and share a common microdomain and are thus concurrently internalized. In the presence of LHbisD4, a larger fraction of VEGFR2 moves from the cell surface to the endocytotic com- 


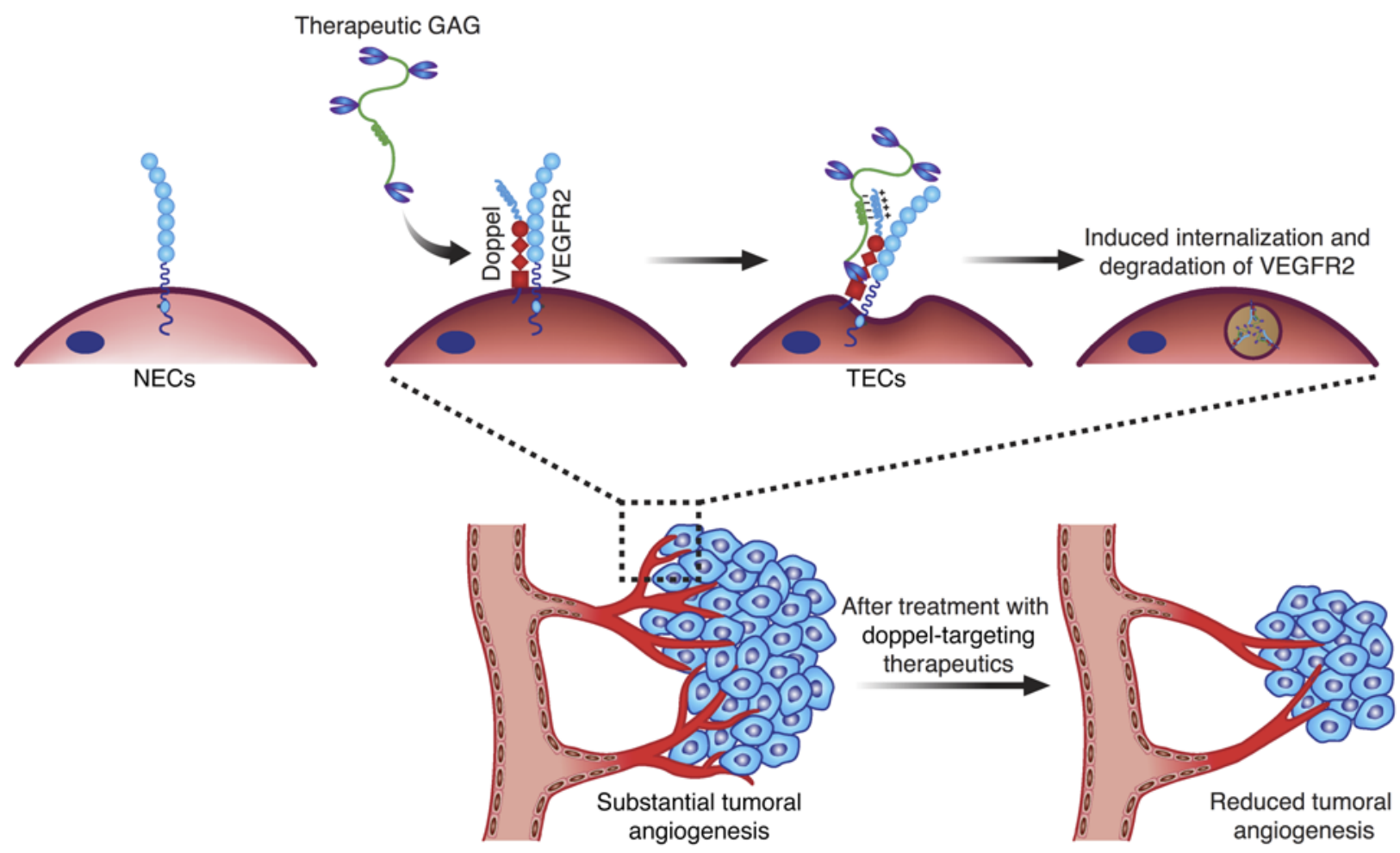

Figure 11. Strategy for targeting doppel-expressing angiogenic tumors. GAG-based therapeutic material binds with the tumor endothelial marker doppel, which constitutively interacts with surface VEGFR2 in TECs, but not NECs. This subsequently triggers induced internalization of the doppel-VEGFR2 complex and inhibits VEGF signaling and angiogenesis in tumors.

partments, with no translocation back to the cell surface. This process therefore keeps surface VEGFR2 in TECs at reduced levels. We speculate that the mechanism by which LHbisD4 or $\alpha$-doppel binds to the doppel/VEGFR2 axis also enhances the endocytosis of VEGFR2. It is also possible that the crosslinking of cell-surface proteins by Abs and other ligands stimulates the internalization of these proteins by TECs (35). Alternatively, sulfated glycans can cause oligomerization and aggregation of bound proteins (36).

\section{Discussion}

We found that doppel was ubiquitously expressed during the angiogenesis that occurred in a variety of tumors. Little is known about the aberrant expression of doppel in TECs or in the early stage of brain endothelial cell development. An intron 1 construct, when inserted into the promoter region of the Prnd gene, enhances doppel expression in ECs, but not in non-ECs (37). Nevertheless, the upstream regulation of doppel expression in TECs needs to be elucidated. This study, in conjunction with previous reports $(6,7)$, demonstrates that doppel regulates tumoral angiogenesis, at least in part, by attenuating VEGFR2 signaling. This study also establishes that doppel selectively regulates angiogenesis in the tumor, but spares normal vasculatures.

Our TMA analysis of human lung and colon cancers revealed that doppel expression increased selectively in tumor-associated vasculatures. Although doppel was largely expressed in tumoral blood vessels, it was not expressed in the quiescent vessels of adjacent cancer tissues or normal organs. Doppel expression and concomitant vessel formation elucidate the proangiogenic roles of doppel in cancer EC biology. To evaluate the proangiogenic functions of doppel, $\mathrm{Hu}^{\text {tluc }}$ cells were stably transfected with doppel and grown in 3D spheroids. Spheroids are genetically more stable than suspended single cells $(38,39)$. ECs that aggregate to form spheroids establish cell-cell contacts and become less apoptotic and more responsive to growth factors. Eventually, the outer monolayers of EC spheroids polarize, differentiate, and become survival factor-independent cells (24). Thus, the implantation of EC spheroids, along with a mixture of survival growth factors (VEGF and basic FGF [bFGF]), builds durable and functional blood vessels in vivo (22). Doppel-mediated development of an aggressive vascular bed in transfected ECs, but not in nontransduced ECs, points to its role in tumor angiogenesis. Since doppel expression was TEC specific, the role of host-derived mural or other endothelial progenitor cells in the formation of a vascular bed was not accounted for. However, whether doppel helps recruit mural or endothelial progenitor cells and facilitates the formation of tumor vessels will require additional studies.

Regulation of downstream signaling of the VEGF/VEGFR2 axis is the main action of this tumoral angiogenesis marker, doppel. Attenuation of the receptor-signaling pathway was specific to VEGFR2 and unaffected by the phosphorylation status of VEGFR1 and VEGFR3. Doppel blocking decreased VEGF-induced phosphorylation of VEGFR2, AKT, ERK1/2, RpS6, and Src. VEGF is a potent inducer of the angiogenic cascades (40), and its receptors are expressed by both TECs and NECs (41). We showed that doppel expression in TECs is the hallmark that distinguishes them from 
NECs. This study, for the first time to our knowledge, also shows that doppel is directly linked with VEGFR2. Next, we attempted to understand how doppel blocking impacts VEGFR2. The endocytosis of VEGFR2 is important for its biological function and signaling capability (42). The amplitude, specificity, and duration of signaling, for example, depend on whether a receptor is removed from the plasma membrane. Doppel blocking simultaneously decreased the membrane content of doppel and VEGFR2. Compared with VEGF, which stimulates endocytosis, degradation, and membrane trafficking of VEGFR2 $(29,43)$, doppel blocking triggered VEGFR2 internalization in a dynamin-dependent manner, whereby it often colocalized with EEA1. In contrast to VEGF stimulation, where fractions of VEGFR2 are recycled back to the membrane - a mechanism that is attributed to Src activation (28) - doppel inhibition triggered lysosomal compartmentalization of VEGFR2. Doppel inhibition affected the total turnover rate of VEGFR2 and its associated signaling pathways; this process was reversed in the presence of a lysosome or endocytosis inhibitor, but not in the presence of a proteasome inhibitor. However, we do not know how doppel prevents the internalization and degradation of VEGFR2. Given the above data, we hypothesize that doppel prolongs the surface residency of VEGFR2 and amplifies the responsiveness of VEGF to VEGFR2. Further studies will be required to clarify how doppel regulates VEGFR2 signaling and the fate of this receptor. Importantly, doppelmediated internalization of VEGFR2 deactivates and prevents the downstream signaling of the receptor. This differential regulation of VEGFR2 endocytosis in TECs caused by doppel inhibition could be a key pathway for controlling angiogenesis in tumors.

Although a number of important targets in TECs and ECs have been identified previously, their therapeutic potential is largely unknown. Moreover, higher expression levels of a protein do not guarantee the protein's applicability as a therapeutic target. This limitation prompted us to determine whether pharmacological inhibition of doppel can selectively abrogate angiogenesis in tumors. Doppel was initially identified as a prion-like protein with a $25 \%$ sequence homology to the normal prion protein $\operatorname{PrP}(12,44$, 45). Doppel is rich in basic amino acids at the N-terminal region, which allows the protein to bind with sulfated GAGs in a fashion similar to that of the binding between PrP and heparan sulfate. Of the diverse sulfated glycans, the use of heparin-like GAG would be a clever approach, because heparin has a good safety profile and is widely used in clinical practice. However, the tissue-targeting ability of heparin is still unknown. To examine the homing ability of heparin, we used TEC spheroids to generate phenotypic, perfusable angiogenic vessels mimicking tumoral blood vessels in vivo. The expression of doppel on TEC surfaces appeared to be crucial for the targeting ability of LMWH, because LMWH nonspecifically bound with $\mathrm{TEC}^{-/- \text {dpl }}$ in vitro and was inefficient at targeting the $\mathrm{TEC}^{-/ \text {-dpl }}$ spheroid plug in vivo. We achieved a 2.3-fold greater ability of LMWH to target TEC spheroids in vivo. Several issues are still unresolved regarding the use heparin as an effective tumor endothelium-targeting agent. Because LMWH is a potent anticoagulant, has a poor affinity for doppel, and requires parenteral injection, we developed orally active heparin-based conjugates for targeting doppel and that could accumulate specifically in TECs.

As therapeutic agents, polysaccharides have several advantages. Polysaccharides exhibit favorable pharmacokinetics, have enhanced tumor-penetrating properties, are stable and structurally heterogeneous, and can be easily modified and synthesized. Because of their complex and nonlinear structure, polysaccharides are less immunogenic and do not become resistant (46). In this study, we have proposed a doppel-targeting therapeutic strategy using bile acid-based LMWH conjugates that absorb orally, target tumor vessels, and attenuate growth factor receptor signaling only at tumor sites. Oral drugs are preferred over injectable drugs, because oral forms can be administered chronically for maintenance therapy. In addition, the Abs used in this study were polyclonal; mAbs are preferred for clinical applications. Doppelspecific mAbs would be ideal for clinical use and should be developed in the future. Conjugation of dimeric DOCA to LMWH produced molecules with enhanced affinity for doppel, but reduced anticoagulant activity. A signature trisulfated pentasaccharide unit of heparin is responsible for the antithrombin III (ATIII) binding (47). We hypothesized that dimeric DOCA derivatives impede the binding between the pentasaccharide unit of LMWH and ATIII and decrease the anticoagulant activity of LMWH $(48,49)$. However, DOCA conjugation increased the binding affinity of LHbisD4doppel by approximately 32-fold. LHbisD4 inhibited VEGFR2 signaling in doppel-expressing ECs, but did not affect its signaling in NECs. PrP is expressed in adult brain endothelium, which is involved in the trans-endothelial migration of monocytes via interaction with adhesion molecules (50). Since LHbisD4 effectively inhibited VEGFR2 signaling in doppel-expressing tumor endothelium, but not in PrP-expressing normal brain endothelium, we concluded that the effect was a direct result of modulating the activity of doppel, but not of PrP. Again, in some neuronal cells or diseases, doppel and $\mathrm{PrP}$ concentrate over the lipid rafts of membranes and remain close to each other $(51,52)$. Although published studies showed upregulation of PrP in tumors $(53,54)$, none showed overexpression or interaction of $\operatorname{PrP}$ and doppel in tumor-specific ECs. Thus, we did not assess the role of PrP in tumor pathogenesis.

LHbisD4 preponderantly homed to tumor because of its superior affinity for doppel. The accumulation of LHbisD4 was 23-fold greater in tumors than in other organs such as the liver. Further, within approximately 1 to 2 hours after oral administration, LHbisD4 showed greater tumor contrast, but low blood contrast. The targeting efficiency of LHbisD4 toward different tumors was proportional to the expression of doppel in the TECs derived from these tumors. Dye-labeled LHbisD4 also showed selectivity for the blood vessels of various tumors. LHbisD4 did not accumulate in the testes, because doppel was not expressed in the blood vessels, although it was expressed in sertoli cells and spermatozoa. The blood-testis barrier may have restricted the passage of LHbisD4, a macromolecule, to the testes $(16,55,56)$. Finally, orally delivered LHbisD 4 was effective in treating 2 mouse models of cancer. In both cases, the degree of angiogenesis, tumor endothelium fraction, and tumor growth was proportionally decreased.

To date, clinical efforts have largely been focused on developing VEGF or direct VEGFR inhibitors. Despite the success with VEGF inhibitors, a number of challenges must be overcome. Although VEGF blocking prevents cancer progression, VEGF targeting induces resistance, causes side effects, and promotes invasion and metastasis $(57,58)$. Our study clearly elucidates that doppel is a clinically important cell-surface candidate for targeted antiangiogenic 
therapy, but more studies are needed to completely define the role of doppel in the growth of tumoral blood vessels. Selective upregulation of doppel in TECs suggests that doppel targeting is unlikely to indiscriminately stifle the signaling pathways throughout the body. Thus, this targeting approach can specifically avoid the side effects associated with VEGF blocking. Given the challenges and varying responses associated with antiangiogenic drugs, a "doppel index" can be developed according to its expression levels in different tumors; this index could be used as a marker for populations of likely responders versus nonresponders. Reports concerning VEGF inhibitor-related metastasis are conflicting; some studies suggest enhanced metastasis in preclinical models $(25,59)$, while others showed no increased metastasis in either preclinical or clinical studies (60-62). VEGF suppresses HGF-dependent c-MET activation, and VEGF blockade restores MET activity, thereby increasing tumor invasiveness (25). Since the doppel inhibitor LHbisD4 targets the doppel/VEGFR2 axis and not the VEGF/VEGFR2 axis, inhibition of doppel can offset the compensatory response elicited by VEGF blocking. To translate this knowledge from bench to bedside, future studies should identify the dose and establish a dosing regimen for patients. On the whole, this study identifies the doppel/VEGFR2 axis as a new molecular target and shows that interruption of this target would bring about more therapeutic benefit than would blocking the physiologically and pathologically important VEGF/VEGFR2 axis.

\section{Methods}

Detailed methodologies are described in the Supplemental Methods.

Human cancer TMAs. Human testes and clinical specimens of lung and colon tumors, including normal tissues and tissues adjacent to tumors, were purchased from US Biomax Inc. For IF staining, TMAs were blocked and stained in the presence of blocking buffers. Doppel was detected with goat (G-20) $\alpha$-doppel and costained with mouse anti-human CD34 Ab. All IF images were captured using a confocal scanning microscope (LSM 710; Carl Zeiss and DM IRB/E; Leica Microsystems) at the National Center for Inter-University Research Facilities (NCIRF, SNU, Korea).

Tumor tissues, cell lines, and animal studies. SCC7, MDAMB-231, A549, CT26, HCT119, СT26, HepG2, and HT29 cells were purchased from the American Type Culture Collection (ATCC). All tumor cell lines were maintained in DMEM medium containing $10 \%$ FBS. Cells $\left(1 \times 10^{6}\right.$ to $1 \times 10^{7}$ cells per mouse $)$ were inoculated s.c. to form tumors. B.End. 3 cells (mouse normal brain ECs), HUVECs, and luciferase-expressing HUVECs were purchased from the ATCC, PromoCell $\mathrm{GmbH}$, and ProQinase $\mathrm{GmbH}$, respectively, and cultured in EC growth medium (ECGM) (PromoCell) with supplement mix that was supplemented with $1 \%$ penicillin-streptomycin (vol/vol). TECs were isolated from different tumors and grown in an optimized culture media to express doppel for an extended period. Details of TEC isolation and culture methods can be found in the Supplemental Methods.

Statistics. Nonparametric and normally distributed data were analyzed by an unpaired, 1-tailed Mann-Whitney $U$ test and a 2-tailed Student's $t$ test, respectively, using GraphPad Prism, version 6.0c (GraphPad Software). $P$ values of less than 0.05 were considered statistically significant. All results are presented as the mean \pm SEM.

Study approval. All animal experiments and surgical procedures were performed according to the regulations of the IACUC of the Seoul National University animal care facility, as described in the Regulation for the Care of Animals (IACUC no. SNU-070822-5) and corresponding to NIH guidelines (Guide for the Care and Use of Laboratory Animals. NIH publication no. 85-23. National Academy Press. 1996). All study participants provided informed consent, and the Seoul National University ethics review board approved the study design.

\section{Author contributions}

TAA conceived, designed, and performed the majority of the experiments and analyzed the data. SWC, JC, FA, and JP assisted with the synthesis and helped plan the study. SWC performed binding affinity studies. JP performed simulation studies. FA and JC helped with in vivo studies. SYK, SWK, FA, ISK, SYK, and YB discussed the results and commented on the manuscript. FA helped with the study design and co-wrote the manuscript with TAA, ISK, and YB.

\section{Acknowledgments}

This study was supported by the Pharosgen Corp. in South Korea. This study was also supported by grants from the Bio \& Medical Technology Development Program (grant 2012028833); the Basic Science Research Program (grant 2010-0027955) of the National Research Foundation (NRF) of Korea; the KIST Institutional Program; the National R\&D Program for Cancer Control; the Ministry of Welfare of the Republic of Korea; and the Korean government (Ministry of Science, ICT, and Future Planning [MSIP]).

Address correspondence to: Youngro Byun, Research Institute of Pharmaceutical Sciences, College of Pharmacy, and Department of Molecular Medicine and Biopharmaceutical Sciences, Graduate School of Convergence Science and Technology, Seoul National University, Building 21, Room 314, 599 Gwanak-gu, Sillim-9-dong, Seoul 151-742, South Korea. Phone: 82.2.880.9525; E-mail: yrbyun@snu.ac.kr. Or to: In-San Kim, Biomedical Research Institute, Korea Institute of Science and Technology, Building L1124, 5 Hwarang-ro 14-gil, Seongbuk-gu, Seoul 136-791, South Korea. Phone: 82.2.958.5935; E-mail: iskim@kist.re.kr.
1. Folkman J. Role of angiogenesis in tumor growth and metastasis. Semin Oncol. 2002;29(6 suppl 16):15-18

2. Cook KM, Figg WD. Angiogenesis inhibitors: current strategies and future prospects. CA Cancer J Clin. 2010;60(4):222-243.

3. Weis SM, Cheresh DA. Tumor angiogenesis: molecular pathways and therapeutic targets. Nat Med. 2011;17(11):1359-1370.

4. van Heeckeren WJ, Ortiz J, Cooney MM, Remick SC. Hypertension, proteinuria, and antagonism of vascular endothelial growth factor signaling: clinical toxicity, therapeutic target, or novel biomarker? J Clin Oncol. 2007;25(21):2993-2995. 5. Dy GK, Adjei AA. Understanding, recognizing, and managing toxicities of targeted anticancer therapies. CA Cancer J Clin. 2013;63(4):249-279.

6. Seaman S, Stevens J, Yang MY, Logsdon D, GraffCherry C, St Croix B. Genes that distinguish physiological and pathological angiogenesis. Cancer Cell. 2007;11(6):539-554.

7. Li A, et al. Physiological expression of the gene for PrP-like protein, PrPLP/Dpl, by brain endothelial cells and its ectopic expression in neurons of PrP-deficient mice ataxic due to Purkinje cell degeneration. Am J Pathol. 2000;157(5):1447-1452.

8. Bueler $\mathrm{H}$, et al. Normal development and behaviour of mice lacking the neuronal cell-surface PrP protein. Nature. 1992;356(6370):577-582.

9. Behrens A, et al. Absence of the prion protein homologue Doppel causes male sterility. EMBOJ. 2002;21(14):3652-3658. 
10. Warner RG, Hundt C, Weiss S, Turnbull JE. Identification of the heparan sulfate binding sites in the cellular prion protein. J Biol Chem. 2002;277(21):18421-18430.

11. Vieira TC, Reynaldo DP, Gomes MP, Almeida MS, Cordeiro Y, Silva JL. Heparin binding by murine recombinant prion protein leads to transient aggregation and formation of RNA-resistant species. JAm Chem Soc. 2011;133(2):334-344.

12. Luhrs T, Riek R, Guntert P, Wuthrich K. NMR structure of the human doppel protein. JMol Biol. 2003;326(5):1549-1557.

13. Kane RS. Thermodynamics of multivalent interactions: influence of the linker. Langmuir. 2010;26(11):8636-8640.

14. Ernst B, Magnani JL. From carbohydrate leads to glycomimetic drugs. Nat Rev Drug Discov. 2009;8(8):661-677.

15. Alam F, et al. Oral delivery of a potent antiangiogenic heparin conjugate by chemical conjugation and physical complexation using deoxycholic acid. Biomaterials. 2014;35(24):6543-6552.

16. Peoc'h K, et al. The human "prion-like" protein Doppel is expressed in both Sertoli cells and spermatozoa. J Biol Chem. 2002;277(45):43071-43078.

17. Rouwkema J, De Boer J, Van Blitterswijk CA. Endothelial cells assemble into a 3-dimensional prevascular network in a bone tissue engineering construct. Tissue Eng. 2006;12(9):2685-2693.

18. Yang JW, et al. Telomerized human microvasculature is functional in vivo. Nat Biotechnol. 2001;19(3):219-224.

19. Wang ZZ, et al. Endothelial cells derived from human embryonic stem cells form durable blood vessels in vivo. Nat Biotechnol. 2007;25(3):317-318.

20. Nor JE, et al. Engineering and characterization of functional human microvessels in immunodeficient mice. Lab Invest. 2001;81(4):453-463.

21. Koike N, Fukumura D, Gralla O, Au P, Schechner JS, Jain RK. Tissue engineering: creation of long-lasting blood vessels. Nature. 2004;428(6979):138-139.

22. Alajati A, et al. Spheroid-based engineering of a human vasculature in mice. Nat Methods. 2008;5(5):439-445.

23. Laib AM, Bartol A, Alajati A, Korff T, Weber H, Augustin HG. Spheroid-based human endothelial cell microvessel formation in vivo. Nat Protoc. 2009;4(8):1202-1215.

24. Korff T, Augustin HG. Integration of endothelial cells in multicellular spheroids prevents apoptosis and induces differentiation. J Cell Biol. 1998;143(5):1341-1352.

25. Lu KV, et al. VEGF inhibits tumor cell invasion and mesenchymal transition through a MET/ VEGFR2 complex. Cancer Cell. 2012;22(1):21-35.

26. Nakayama M, et al. Spatial regulation of VEGF receptor endocytosis in angiogenesis. Nat Cell Biol. 2013;15(3):249-260.

27. Nakamura Y, et al. Role of protein tyrosine phosphatase $1 \mathrm{~B}$ in vascular endothelial growth factor signaling and cell-cell adhesions in endothelial cells. Circ Res. 2008;102(10):1182-1191.

28. Gampel A, Moss L, Jones MC, Brunton V, Norman JC, Mellor H. VEGF regulates the mobilization of VEGFR2/KDR from an intracel- lular endothelial storage compartment. Blood. 2006;108(8):2624-2631.

29. Bruns AF, et al. Ligand-stimulated VEGFR2 signaling is regulated by co-ordinated trafficking and proteolysis. Traffic. 2010;11(1):161-174.

30. Meyer RD, Srinivasan S, Singh AJ, Mahoney JE, Gharahassanlou KR, Rahimi N. PEST motif serine and tyrosine phosphorylation controls vascular endothelial growth factor receptor 2 stability and downregulation. Mol Cell Biol. 2011;31(10):2010-2025.

31. Shyng SL, Lehmann S, Moulder KL, Harris DA. Sulfated glycans stimulate endocytosis of the cellular isoform of the prion protein, PrPC, in cultured cells. J Biol Chem. 1995;270(50):30221-30229.

32. Al-Hilal TA, Alam F, Byun Y. Oral drug delivery systems using chemical conjugates or physical complexes. Adv Drug Deliv Rev. 2013;65(6):845-864.

33. Al-Hilal TA, et al. Oligomeric bile acid-mediated oral delivery of low molecular weight heparin. J Control Release. 2014;175:17-24.

34. Al-Hilal TA, et al. Functional transformations of bile acid transporters induced by high-affinity macromolecules. Sci Rep. 2014;4:4163.

35. Marsh EW, Leopold PL, Jones NL, Maxfield FR. Oligomerized transferrin receptors are selectively retained by a lumenal sorting signal in a long-lived endocytic recycling compartment. JCell Biol. 1995;129(6):1509-1522.

36. Jackson RL, Busch SJ, Cardin AD. Glycosaminoglycans: molecular properties, protein interactions, and role in physiological processes. Physiol Rev. 1991;71(2):481-539.

37. Nagyova J, Pastorek J, Kopacek J. Identification of the critical cis-acting elements in the promoter of the mouse Prnd gene coding for Doppel protein. Biochim Biophys Acta. 2004;1679(3):288-293.

38. Bates RC, Buret A, van Helden DF, Horton MA, Burns GF. Apoptosis induced by inhibition of intercellular contact. JCell Biol. 1994;125(2):403-415.

39. Lincz LF, Buret A, Burns GF. Formation of spheroid structures in a human colon carcinoma cell line involves a complex series of intercellular rearrangements. Differentiation. 1997;61(4):261-274.

40. Ferrara N. VEGF and the quest for tumour angiogenesis factors. Nat Rev Cancer. 2002;2(10):795-803.

41. Smith NR, et al. Vascular endothelial growth factor receptors VEGFR-2 and VEGFR-3 are localized primarily to the vasculature in human primary solid cancers. Clin Cancer Res. 2010;16(14):3548-3561.

42. Simons M. An inside view: VEGF receptor trafficking and signaling. Physiology. 2012;27(4):213-222.

43. Manickam V, et al. Regulation of vascular endothelial growth factor receptor 2 trafficking and angiogenesis by Golgi localized t-SNARE syntaxin 6. Blood. 2011;117(4):1425-1435.

44. Moore RC, et al. Ataxia in prion protein (PrP)-deficient mice is associated with upregulation of the novel PrP-like protein doppel. J Mol Biol. 1999;292(4):797-817.

45. Moore RC, et al. Doppel-induced cerebellar degeneration in transgenic mice. Proc Natl Acad Sci U S A. 2001;98(26):15288-15293.

46. Shriver Z, Raguram S, Sasisekharan R. Gly- comics: a pathway to a class of new and improved therapeutics. Nat Rev Drug Discov. 2004;3(10):863-873.

47. Weitz JI. Low-molecular-weight heparins. N Engl JMed.1997;337(10):688-698.

48. Park JW, et al. High antiangiogenic and low anticoagulant efficacy of orally active low molecular weight heparin derivatives. J Control Release. 2010;148(3):317-326.

49. Chung SW, et al. Potentiation of anti-angiogenic activity of heparin by blocking the ATIII-interacting pentasaccharide unit and increasing net anionic charge. Biomaterials. 2012;33(35):9070-9079.

50. Viegas P, Chaverot N, Enslen H, Perriere N, Couraud PO, Cazaubon S. Junctional expression of the prion protein PrPC by brain endothelial cells: a role in trans-endothelial migration of human monocytes. J Cell Sci. 2006;119(pt 22):4634-4643.

51. Massimino ML, et al. Human Doppel and prion protein share common membrane microdomains and internalization pathways. Int J Biochem Cell Biol. 2004;36(10):2016-2031.

52. Watts JC, et al. Interactome analyses identify ties of PrP and its mammalian paralogs to oligomannosidic N-glycans and endoplasmic reticulum-derived chaperones. PLoS Pathog. 2009;5(10):e1000608.

53. de Wit M, et al. Cell surface proteomics identifies glucose transporter type 1 and prion protein as candidate biomarkers for colorectal adenoma-to-carcinoma progression. Gut. 2012;61(6):855-864.

54. Pan Y, et al. Cellular prion protein promotes invasion and metastasis of gastric cancer. FASEB J. 2006;20(11):1886-1888.

55. Su L, Mruk DD, Cheng CY. Drug transporters, the blood-testis barrier, and spermatogenesis. J Endocrinol. 2011;208(3):207-223.

56. Bany BM, Hamilton GS. Assessment of permeability barriers to macromolecules in the rodent endometrium at the onset of implantation. Methods Mol Biol. 2011;763:83-94.

57. Shojaei F. Anti-angiogenesis therapy in cancer: current challenges and future perspectives. Cancer Lett. 2012;320(2):130-137.

58. Welti J, Loges S, Dimmeler S, Carmeliet P. Recent molecular discoveries in angiogenesis and antiangiogenic therapies in cancer. JClin Invest. 2013;123(8):3190-3200.

59. Ebos JM, Lee CR, Cruz-Munoz W, Bjarnason GA, Christensen JG, Kerbel RS. Accelerated metastasis after short-term treatment with a potent inhibitor of tumor angiogenesis. Cancer Cell. 2009;15(3):232-239.

60. Padera TP, et al. Differential response of primary tumor versus lymphatic metastasis to VEGFR-2 and VEGFR-3 kinase inhibitors cediranib and vandetanib. Mol Cancer Ther. 2008;7(8):2272-2279.

61. Singh M, et al. Anti-VEGF antibody therapy does not promote metastasis in genetically engineered mouse tumour models. J Pathol. 2012;227(4):417-430.

62. Miles D, et al. Disease course patterns after discontinuation of bevacizumab: pooled analysis of randomized phase III trials. J Clin Oncol. 2011;29(1):83-88. 